\title{
INTEGRAL FIELD SPECTROSCOPY OF THE LUMINOUS INFRARED GALAXY ARP 299 (IC $694+$ NGC 3690) ${ }^{1}$
}

\author{
M. García-Marín, ${ }^{2}$ L. Colina, ${ }^{2}$ S. Arribas, ${ }^{3,4,5}$ A. Alonso-Herrero, ${ }^{2}$ and E. Mediavilla ${ }^{5}$ \\ Received 2006 March 14; accepted 2006 June 20
}

\begin{abstract}
The luminous infrared galaxy Arp 299 (IC 694 + NGC 3690) is studied using optical integral field spectroscopy obtained with the INTEGRAL system, together with archival Hubble Space Telescope WFPC2 and NICMOS images. The stellar and ionized gas morphology shows $\lambda$-dependent variations due to the combined effects of the dust internal extinction and the nature and spatial distribution of the different ionizing sources. The two-dimensional ionization maps have revealed an off-nuclear conical structure of about $4 \mathrm{kpc}$ in length, characterized by highexcitation conditions and a radial gradient in the gas electron density. The apex of this structure coincides with B1 region of NGC 3690, which in turn presents Seyfert-like ionization, high extinction, and a high velocity dispersion. These results strongly support the hypothesis that B1 is the true nucleus of NGC 3690, where an AGN is located. In the circumnuclear regions $\mathrm{H}$ II-like ionization dominates, while LINER-like ionization is found elsewhere. The $\mathrm{H} \alpha$-emitting sources with ages from 3.3 to $7.2 \times 10^{6} \mathrm{yr}$, have masses of between 6 and $680 \times 10^{6} M_{\odot}$ and contribute about $45 \%$ to the bolometric luminosity (extinction corrected). The ionized ( $\mathrm{H} \alpha)$ and neutral (Na D) gas velocity fields show similar structure on scales of several hundred to about $1 \mathrm{kpc}$, indicating that these gas components are kinematically coupled. The kinematic structure is complex and on scales of about $0.2 \mathrm{kpc}$ does not appear to be dominated by the presence of ordered, rotational motions. The large velocity dispersion measured in NGC 3690 indicates that this galaxy is the most massive of the system. The low velocity amplitude and dispersion of the interface suggest that the ionized gas is slowly rotating or in a close to quiescent phase.
\end{abstract}

Subject headings: galaxies: individual (Arp 299, IC 694, NGC 3690) — galaxies: interactions galaxies: starburst — galaxies: nuclei — galaxies: stellar content galaxies: kinematics and dynamics - infrared: galaxies

Online Material: color figures

\section{INTRODUCTION}

Arp 299 is a nearby luminous infrared galaxy $(d \sim 43 \mathrm{Mpc}$ for $\left.H_{0}=70 \mathrm{~km} \mathrm{~s}^{-1} \mathrm{Mpc}^{-1}, \Omega_{M}=0.7, \Omega_{\Lambda}=0.3\right)$ with an infrared $(8-1000 \mu \mathrm{m})$ luminosity of $L_{\mathrm{IR}}=5.7 \times 10^{11} L_{\odot}{ }^{6}$ It is an interacting system (Fig. 1) composed of two individual galaxies (IC 694 and NGC $3690^{7}$ ) in an early dynamical stage. Hibbard \& Yun (1999) have discovered an $\mathrm{H}$ I tidal tail of $180 \mathrm{kpc}$ in length, with no evidence for tidal dwarf galaxies. Powerful starburst regions with star formation rates of up to about $100 M_{\odot} \mathrm{yr}^{-1}$ have been identified (Alonso-Herrero et al. 2000, hereafter AAH00). Moreover, the system presents several regions where supernova remnants have been detected at radio wavelengths (Neff et al.

\footnotetext{
${ }^{1}$ Based on observations with the William Herschel Telescope operated on the island of La Palma by the ING in the Spanish Observatorio del Roque de los Muchachos of the Instituto de Astrofísica de Canarias. Based also on observations with the NASA-ESA Hubble Space Telescope, obtained at the Space Telescope and Science Institute, which is operated by the Association of Universities for Research in Astronomy, Inc. under NASA contract number NAS5-26555.

${ }_{2}$ Departamento de Astrof ísica Molecular e Infrarroja, Instituto de Estructura de la Materia, CSIC, E-28006 Madrid, Spain.

3 Space Telescope Science Institute, 3700 San Martin Drive, Baltimore, MD 21218 .

${ }^{4}$ On leave from the Instituto de Astrof ísica de Canarias (IAC) and from the Consejo Superior de Investigaciones Científicas (CSIC), Spain.

5 Instituto de Astrofísica de Canarias ( IAC), 38205 La Laguna, Canary Islands, Spain.

${ }^{6}$ IRAS fluxes obtained from Moshir et al. (Vizier Online Data Catalog II/156A, 1993). $L_{\mathrm{IR}}$ derived from the equation detailed on Sanders \& Mirabel (1996).

7 Through this paper, we use the notation introduced by Gehrz et al. (1983) for the two components of Arp 299: the nucleus of IC 694 (eastern component) is region A, while the main sources in NGC 3690 (western component) are B1, B2, $\mathrm{C}\left(\mathrm{C} 1+\mathrm{C} 2+\mathrm{C} 3\right.$ ), and $\mathrm{C}^{\prime}$ (see also Alonso-Herrero et al. 2000).
}

2004). More recently, Mattila et al. (2005) have discovered a new supernova using near-IR images, reinforcing the prediction that this galaxy has a high rate of supernova explosions (Mannucci et al. 2003).

Optical spectroscopic studies have classified IC 694 as starburst and NGC 3690 as starburst/LINER (e.g., Coziol et al. 1998), and mid-IR observations classified the system as starburst (e.g., Laurent et al. 2000). Using Hubble Space Telescope (HST) imaging and near-infrared spectroscopy, AAH00 modeled the star formation properties of the system without the need for an active galactic nucleus (AGN). The first hint for the presence of an AGN in Arp 299 came from X-ray observations with the BeppoSAX satellite by Della Ceca et al. (2002). These authors concluded that a highly absorbed $\left(N_{\mathrm{H}} \simeq 2.5 \times 10^{24} \mathrm{~cm}^{-2}\right)$ AGN, with an intrinsic luminosity of $L_{0.5-100 \mathrm{keV}} \simeq 1.9 \times 10^{43} \mathrm{ergs} \mathrm{s}^{-1}$, is present in this galaxy, although due to the low spatial resolution its exact location could not be determined. Using Chandra and XMMNewton observations, recent studies (Ballo et al. 2004) have inferred the presence of two AGNs, one located in NGC 3690 (source B1; $6.4 \mathrm{keV}$ line), and another in IC 694 (source A; $6.7 \mathrm{keV} \mathrm{Fe}-\mathrm{H} \alpha$ line). In addition, Gallais et al. (2004) suggest the presence of an AGN in NGC 3690 based on the shape of the mid-IR continuum, while in IC 694 they only detect spectral features characteristic of massive, dust-enshrouded star formation.

This paper presents a detailed study of the two-dimensional star formation, kinematics, and ionized gas properties of Arp 299, covering the two individual galaxies of the system and the interface area. At a distance of about $43 \mathrm{Mpc}$, Arp 299 is one of the nearest interacting, luminous infrared galaxies, and therefore these observations provide high spatial resolution (about $0.205 \mathrm{kpc} \operatorname{arcsec}^{-1}$ ) and allow us to investigate in detail the 


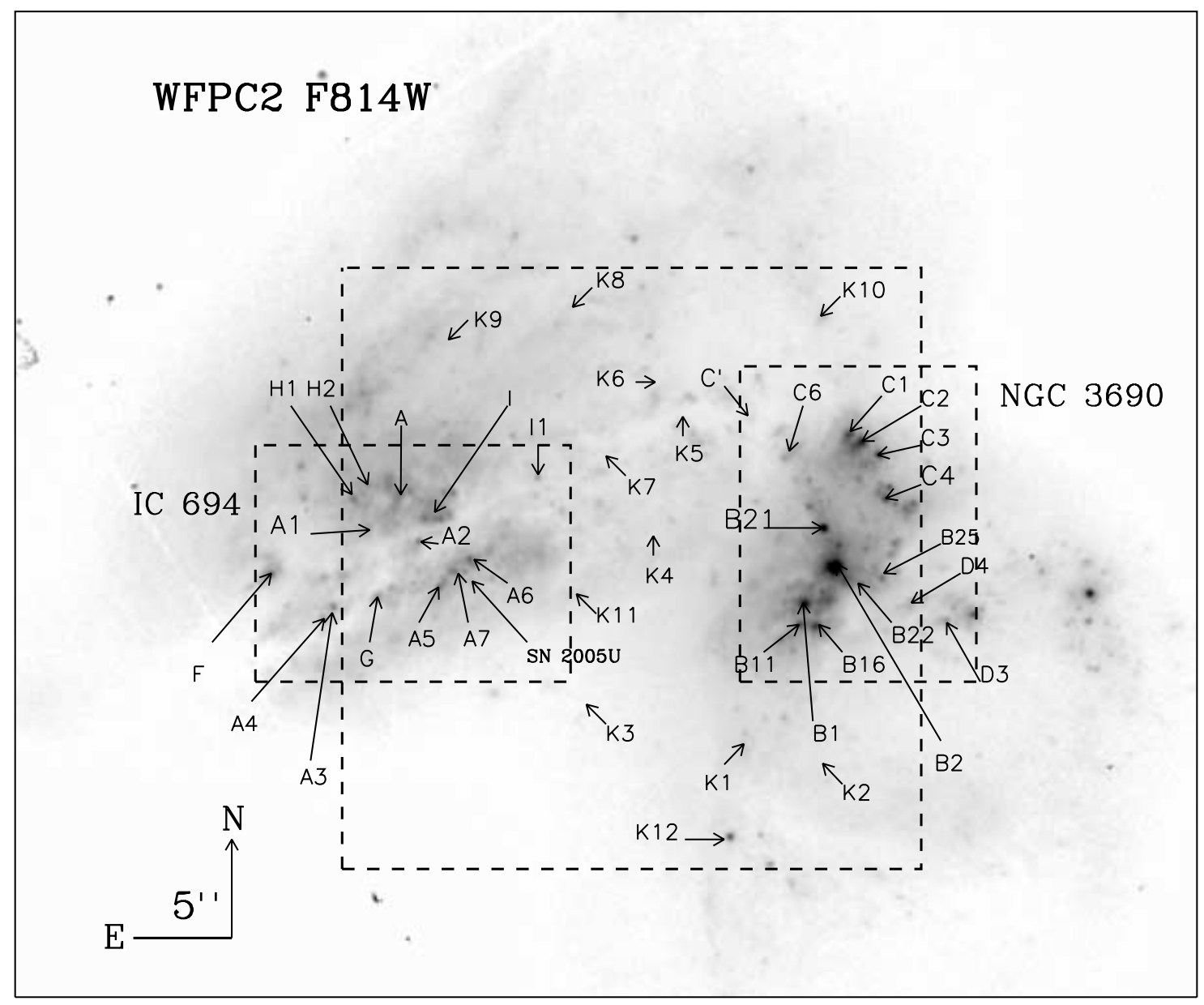

FIG. 1.- Archival HST image of the interacting system Arp 299 obtained with the WFPC2 F814W filter. The rectangular overlays indicate the effective area covered by the three pointings with the two INTEGRAL configurations used (i.e., SB2, small rectangles; SB3, large rectangle). We have marked the positions of the regions under study (see $\S 3$ and Tables 1, 2, and 3). The location of the recently discovered near-IR SN is shown too (Mattila et al. 2005). The image is shown on a logarithmic scale. [See the electronic edition of the Journal for a color version of this figure.]

structure and physical properties of this galaxy. The paper is organized as follows. Section 2 describes the observations and data reduction, while the selection of regions under study is described in $\S 3$. The next sections present a discussion of the results, with special emphasis on the stellar and ionized gas morphology $(\S 4)$, the internal dust distribution and extinction effects $(\S 5)$, the extinction estimates using optical and near-IR emission lines $(\S 5.4)$, the two-dimensional ionization structure $(\S 6)$, the electron density ( $\S 7)$, the origin of the LINER-like ionization $(\S 8)$, the star-forming properties $(\S 9)$, and the kinematics and dynamical mass derivations $(\S 10)$. Finally, $\S 11$ presents a short summary of the main new results.

\section{OBSERVATIONS AND DATA REDUCTION}

Integral field spectroscopy (IFS) of Arp 299 was obtained on 2004 January 15 and 17 using INTEGRAL, a fiber-based integral field system (Arribas et al. 1998) connected to the Wide Field Fibre Optic Spectrograph (WYFFOS; Bingham et al. 1994) and mounted on the $4.2 \mathrm{~m}$ William Herschel Telescope. Two different INTEGRAL configurations were used for observing the system (see arrows in Fig. 1). The fiber bundle with the largest field of view (FOV), SB3, was centered on a position equidistant from the centers of the galaxies and therefore used to map the diffuse, interface region between the two galaxies (IC 694 and NGC 3690) that form the Arp 299 system, as well as the galaxy external re- gions at distances of about $4 \mathrm{kpc}$. The SB3 bundle consists of 115 fibers each 2 ".7 in diameter, covering a rectangular area of 33 ." $6 \times$ 29 ". 4 on the sky. In addition 20 fibers, forming a circle of $45^{\prime \prime} 0$ in radius, were used to obtain a spectrum of the sky simultaneously. The SB2 bundle was used to map each of the two galaxies (IC 694 and NGC 3690, the eastern and western galaxies, respectively in Fig. 1) individually. It consists of 189 fibers each 0.9 in diameter, covering a rectangular area of $16^{\prime \prime} .0 \times 12$ ". 3 on the sky. In addition 30 fibers, forming a circle of 45 ." 0 in radius, were used to measure the sky simultaneously.

The spectra were taken using a 600 lines $\mathrm{mm}^{-1}$ grating, with an effective spectral resolution (FWHM) of approximately 6.0 and $9.8 \AA$ for the SB2 and SB3 bundles, respectively, and covering the spectral range $\lambda \lambda 4400-7000 \AA$. The total integration time was $3600 \mathrm{~s}$ for each pointing with the SB2 bundle, split into three individual exposures of $1200 \mathrm{~s}$ each. The total integration time with the SB3 bundle was 5400 s divided into three individual exposures of $1800 \mathrm{~s}$ each. The seeing conditions were fairly stable during the two periods of observation (about 0 ."7 the first night, when we used the SB2 bundle, and 1"1 the second night when we used the SB3 bundle).

The data reduction consists of three main steps: (1) the basic reduction of two-dimensional fiber spectra, (2) the line fitting, and (3) the generation of maps of spectral features (such as intensity maps and velocity fields) from the calibrated spectra. The 


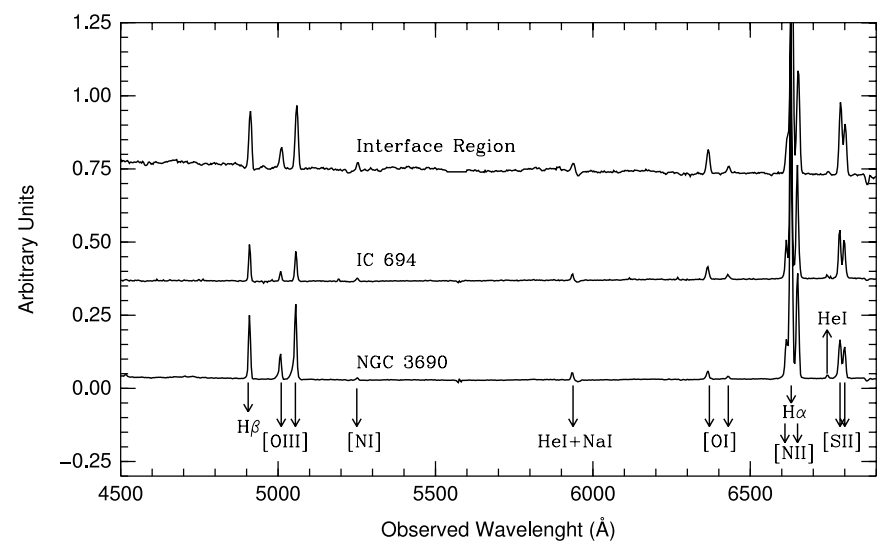

FIG. 2.-Average spectra of the interface region (K1-K12), IC 694 (A, H1H2), and NGC 3690 (B1-B11). The spectral range shown is 4500-6900 A. See Fig. 1 and Tables 1, 2, and 3 for location of the individual emitting sources.

basic reduction was performed using the $\mathrm{IRAF}^{8}$ environment, and followed the standard procedures applied to spectra obtained with two-dimensional fiber spectrographs (Arribas et al. 1997 and references therein). The absolute flux calibration was performed observing the standard star Feige 34 with the same instrument configuration and data reduction procedures used on the galaxy. For each spectrum, the total flux, the radial velocities, and the velocity dispersions $(\sigma)$ were measured by fitting single Gaussian functions to the observed emission-line profiles using the DIPSO package (Howarth \& Murray 1988). For the generation of the maps, a two-dimensional interpolation was applied using the free software IDA (García-Lorenzo et al. 2002). The blue and red stellar continuum images were taken as an emission-line-free filter centered at $\lambda_{\text {rest }}=4475$ and $6525 \AA$, respectively, and with

\footnotetext{
${ }^{8}$ IRAF software is distributed by the National Optical Astronomy Observatory (NOAO), which is operated by the Association of Universities for Research in Astronomy (AURA), Inc., in cooperation with the National Science Foundation.
}

a rectangular bandwidth of $150 \AA$. The measures on the SB3 maps were done through an aperture of 3."0 in diameter, while for the SB2 maps the apertures diameter were 1".0 (IC 694) and 0".9 (NGC 3690, to avoid the overlap between regions).

Three sample spectra, covering the entire used spectral range, are shown in Figure 2. All the spectra show the strong $(\mathrm{H} \beta,[\mathrm{O}$ III] $\lambda \lambda 4959,5007, \mathrm{H} \alpha+[\mathrm{N}$ II] $\lambda \lambda 6548,6584, \mathrm{He}$ I $\lambda 6678$, and [S II] $\lambda \lambda 6716,6731)$ and the weak ( $\mathrm{N}_{\mathrm{I}} \lambda 5199$, , He I $\lambda 5876$, , [O I ] $\lambda \lambda 6300$, 6364 , and $\mathrm{He}$ I 26678 ) emission lines in different ratios, as well as interstellar absorption lines ( $\mathrm{Na}$ I $\lambda \lambda 5890,5896)$, that will be used to trace the neutral interstellar medium kinematics.

Complementary HST multiwavelength images both in continuum and emission lines are also available. These additional data include the red (WFPC2 $-\mathrm{F} 814 \mathrm{~W}$, archive images) and the near-infrared (NICMOS1 - F110M, NICMOS2 - F160W, and NICMOS2 - F222M, from AAH00) continuum images. These filters are the HST equivalent to the ground-based JohnsonCousins system $I, J, H$, and $K$, respectively (Origlia \& Leitherer $2000)$. Images of the ionized gas as traced by the $\operatorname{Pa} \alpha\left(\lambda_{\text {rest }}=\right.$ $1.875 \mu \mathrm{m}$ ) light (NICMOS - F190N, from AAH00) will be used too. The archival WFPC2 image was calibrated on the fly with the best available reference files, while the NICMOS images were reduced with routines from the package NicRed (McLeod 1997; see details on AAH00).

\section{SELECTION OF REGIONS UNDER STUDY}

In order to analyze the system we have selected several regions distributed over the individual galaxies, the interface between them and regions external to the galaxies (Fig. 1). The selection includes compact unresolved high surface brightness knots, star clusters, and more diffuse, extended, resolved low surface brightness areas. For IC 694 and NGC 3690 we based our selection on that done by AAH00, which includes the nuclear regions (A and B1, respectively) and the most important star-forming regions and bright star clusters. To identify the position of the regions under study in the lower resolution INTEGRAL maps, a direct comparison of the convolved WFPC2 image with the red continuum image was performed. The following sections present the main results

TABLE 1

Properties of the Regions of the IC 694 and NGC 3690 Interface

\begin{tabular}{|c|c|c|c|c|c|c|c|c|c|}
\hline Region & $\begin{array}{c}\text { Distance }^{\mathrm{a}} \\
(\mathrm{kpc})\end{array}$ & $\begin{array}{c}\Delta \alpha^{\mathrm{a}} \\
(\operatorname{arcsec})\end{array}$ & $\begin{array}{c}\Delta \delta^{\mathrm{a}} \\
(\operatorname{arcsec})\end{array}$ & $F_{\text {obs }}(\mathrm{H} \alpha)^{\mathrm{b}}$ & $\begin{array}{l}A_{V}{ }^{\mathrm{c}} \\
(\mathrm{mag})\end{array}$ & $\log \left(\left[\begin{array}{ll}\mathrm{III}\end{array}\right] / \mathrm{H} \beta\right)^{\mathrm{d}}$ & $\log \left(\left[\mathrm{O}_{\mathrm{III}} / \mathrm{H} \alpha\right)^{\mathrm{e}}\right.$ & $\log \left(\left[\mathrm{N}_{\mathrm{II}}\right] / \mathrm{H} \alpha\right)^{\mathrm{f}}$ & $\log \left(\left[\mathrm{S}_{\mathrm{II}}\right] / \mathrm{H} \alpha\right)^{\mathrm{g}}$ \\
\hline $\mathrm{K} 1$ & 2.97 & -17.6 & -12.7 & 1.7 & 2.2 & 0.27 & -1.07 & -0.34 & -0.37 \\
\hline K3 & 1.28 & -9.6 & -10.7 & 0.6 & 2.0 & 0.22 & -0.59 & -0.21 & -0.25 \\
\hline 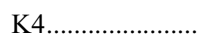 & 2.49 & -13.0 & -1.8 & 2.7 & 1.6 & 0.04 & -0.83 & -0.41 & -0.31 \\
\hline K5 & 3.53 & -14.5 & 3.9 & 10.7 & 2.9 & 0.14 & -1.44 & -0.54 & -0.64 \\
\hline $\mathrm{K} 8$ & 4.07 & -9.0 & 9.8 & 1.4 & 2.7 & 0.14 & -0.71 & -0.32 & -0.23 \\
\hline 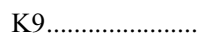 & 3.52 & -2.6 & 7.8 & 3.3 & 1.8 & -0.01 & -0.92 & -0.37 & -0.36 \\
\hline 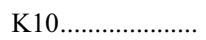 & 5.26 & -21.5 & -9.0 & 4.2 & 1.0 & 0.17 & -1.36 & -0.53 & -0.56 \\
\hline K11 ...................... & 1.44 & -9.1 & -5.1 & 1.6 & 1.5 & 0.02 & -0.82 & -0.39 & -0.31 \\
\hline 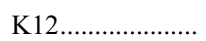 & 3.18 & -16.8 & -17.4 & 7.6 & 2.0 & 0.13 & -0.98 & -0.35 & -0.37 \\
\hline
\end{tabular}

\footnotetext{
a Distance relative to nucleus A.

b Flux measured $\left(\times 10^{-14} \mathrm{ergs} \mathrm{s}^{-1} \mathrm{~cm}^{-2}\right)$ using a circular aperture of 3." Uncertainties $10 \%-15 \%$.

${ }^{c}$ Internal extinction derived from the $\mathrm{H} \alpha / \mathrm{H} \beta$ ratio. Average error in $A_{V}$ is 0.7 mag.

d Average error \pm 0.11 .

e Average error \pm 0.06

${ }^{\mathrm{f}}$ Average error \pm 0.05 .

g Average error \pm 0.06 . In all cases, emission-line ratios are derived using extinction-corrected flux values.
} 
TABLE 2

Properties of the Regions of IC 694

\begin{tabular}{|c|c|c|c|c|c|c|c|c|c|}
\hline Region & $\begin{array}{l}\text { Distance }^{\mathrm{a}} \\
\quad(\mathrm{kpc})\end{array}$ & $\begin{array}{c}\Delta \alpha^{\mathrm{a}} \\
(\operatorname{arcsec})\end{array}$ & $\begin{array}{c}\Delta \delta^{\mathrm{a}} \\
(\operatorname{arcsec})\end{array}$ & $F_{\text {obs }}(\mathrm{H} \alpha)^{\mathrm{b}}$ & $\begin{array}{l}A_{V}^{\mathrm{c}} \\
(\mathrm{mag})\end{array}$ & $\log \left(\left[\mathrm{O}_{\mathrm{III}}\right] / \mathrm{H} \beta\right)^{\mathrm{d}}$ & $\log \left(\left[\mathrm{O}_{\mathrm{I}}\right] / \mathrm{H} \alpha\right)^{\mathrm{e}}$ & $\log \left(\left[\mathrm{N} \mathrm{II}^{\mathrm{II}}\right] / \mathrm{H} \alpha\right)^{\mathrm{f}}$ & $\log ([\mathrm{S} \mathrm{II}] / \mathrm{H} \alpha)^{\mathrm{g}}$ \\
\hline А & 0.00 & 0.0 & 0.0 & 2.9 & 2.9 & -0.14 & -1.03 & -0.31 & -0.41 \\
\hline A2 ..................... & 0.54 & -1.0 & -2.4 & 3.1 & 2.0 & -0.14 & -1.51 & -0.52 & -0.60 \\
\hline 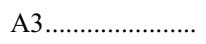 & 1.37 & 3.3 & -5.7 & 2.3 & 1.9 & -0.03 & -1.61 & -0.53 & -0.59 \\
\hline 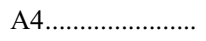 & 1.51 & 3.8 & -6.3 & 3.0 & 2.1 & -0.02 & -1.73 & -0.55 & -0.66 \\
\hline A7 & 0.99 & -2.8 & -3.9 & 6.2 & 2.3 & 0.09 & -1.61 & -0.56 & -0.66 \\
\hline F & 1.58 & 6.5 & -4.1 & 6.7 & 3.2 & 0.10 & -2.06 & -0.52 & -0.92 \\
\hline $\mathrm{G}$ & 1.10 & 1.1 & -5.2 & 6.2 & 1.9 & 0.05 & -1.84 & -0.54 & -0.74 \\
\hline $\mathrm{H} 1$ & 0.48 & 2.3 & -0.3 & 6.4 & 2.8 & -0.15 & -1.41 & -0.47 & -0.63 \\
\hline $\mathrm{H} 2 \ldots \ldots \ldots \ldots \ldots$ & 0.34 & 1.6 & 0.5 & 4.7 & 3.4 & -0.06 & -0.99 & -0.32 & -0.46 \\
\hline I . & 0.42 & -1.8 & -0.9 & 6.0 & 1.6 & -0.09 & -1.60 & -0.52 & -0.65 \\
\hline I1 ............................. & 1.45 & -7.0 & 0.8 & 0.8 & 2.4 & -0.10 & -1.28 & -0.43 & -0.42 \\
\hline
\end{tabular}

${ }^{\mathrm{a}}$ Distance relative to nucleus A.

b Flux measured $\left(\times 10^{-14}\right.$ ergs s $\left.\mathrm{cm}^{-1}\right)$ using a circular aperture of 1 ". Uncertainties $10 \%-15 \%$.

${ }^{c}$ Internal extinction derived from the $\mathrm{H} \alpha / \mathrm{H} \beta$ ratio. Error in $A_{V}$ is $0.5 \mathrm{mag}$.

d Average error \pm 0.08 .

e Average error \pm 0.04 .

${ }^{\mathrm{f}}$ Average error \pm 0.05 .

g Average error \pm 0.04 . In all cases, emission-line ratios are derived using extinction-corrected flux values.

obtained for these different regions and discuss their implications separately for the two individual galaxies, IC 694 and NGC 3690, and for the interface region between them. The physical properties measured for each individual region are presented in Tables $1-3$.

\section{STELLAR AND IONIZED GAS MORPHOLOGY}

Arp 299 is the result of a merger between the galaxies IC 694 and NGC 3690, whose nuclei (A and B1, respectively, see Fig. 3) are located about $4.6 \mathrm{kpc}$ apart. The system shows a morphologically disturbed structure characterized by the presence of compact high surface knots, and a complex system of filaments and dust lanes, as already indicated by previous studies at several wavelengths (e.g., Gehrz et al. 1983; Telesco et al. 1985; Baan \& Haschick 1990; AAH00). In what follows, we discuss the differences in the spatial distribution of the stellar and ionized gas components.

\subsection{Interface Region}

The two INTEGRAL/SB3 continuum images of Arp 299, obtained at wavelengths blueward of $\mathrm{H} \alpha$ and $\mathrm{H} \beta$, reproduce well the overall stellar morphology detected with the HST WFPC2 F814W image (Fig. 3) although at a lower spatial resolution (fiber

TABLE 3

Properties of the Regions of NGC 3690

\begin{tabular}{|c|c|c|c|c|c|c|c|c|c|}
\hline Region & $\begin{array}{l}\text { Distance }^{\mathrm{a}} \\
\quad(\mathrm{kpc})\end{array}$ & $\begin{array}{c}\Delta \alpha^{\mathrm{a}} \\
(\operatorname{arcsec})\end{array}$ & $\begin{array}{c}\Delta \delta^{\mathrm{a}} \\
(\operatorname{arcsec})\end{array}$ & $F_{\text {obs }}(\mathrm{H} \alpha)^{\mathrm{b}}$ & $\begin{array}{l}A_{V}{ }^{\mathrm{c}} \\
(\mathrm{mag})\end{array}$ & $\log \left(\left[\begin{array}{ll}\mathrm{III}\end{array}\right] / \mathrm{H} \beta\right)^{\mathrm{d}}$ & $\log \left(\left[\mathrm{O}_{\mathrm{I}}\right] / \mathrm{H} \alpha\right)^{\mathrm{d}}$ & $\log \left(\left[\mathrm{N}^{\mathrm{II}}\right] / \mathrm{H} \alpha\right)^{\mathrm{e}}$ & $\log \left(\left[\mathrm{S}_{\Pi]}\right] / \mathrm{H} \alpha\right)^{\mathrm{f}}$ \\
\hline B1 & 0.00 & 0.0 & 0.0 & 9.7 & 3.7 & 0.55 & -1.07 & -0.29 & -0.54 \\
\hline 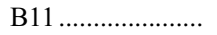 & 0.22 & 0.27 & -1.0 & 5.7 & 2.0 & 0.10 & -1.37 & -0.41 & -0.52 \\
\hline 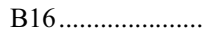 & 0.26 & -0.5 & -1.1 & 10.6 & 1.1 & -0.02 & -1.68 & -0.47 & -0.71 \\
\hline 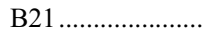 & 0.82 & -1.0 & 3.9 & 3.1 & 2.6 & 0.02 & -1.54 & -0.48 & -0.77 \\
\hline $\mathrm{C} 1 \ldots \ldots \ldots \ldots$ & 1.83 & -2.4 & 8.6 & 28.1 & 3.0 & 0.13 & -1.87 & -0.49 & -0.96 \\
\hline $\mathrm{C} 2 \ldots \ldots \ldots \ldots \ldots \ldots$ & 1.80 & -3.0 & 8.2 & 33.4 & 2.1 & 0.11 & -1.92 & -0.46 & -0.94 \\
\hline 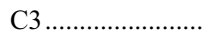 & 1.75 & -3.9 & 7.6 & 16.7 & 1.0 & 0.01 & -1.80 & -0.44 & -0.78 \\
\hline $\mathrm{C} 4 \ldots \ldots \ldots \ldots \ldots$ & 1.39 & -4.2 & 5.3 & 5.7 & 0.5 & -0.03 & -1.80 & -0.47 & -0.67 \\
\hline С6 ......................... & 1.55 & 1.0 & 7.5 & 5.2 & 1.9 & 0.02 & -1.74 & -0.53 & -0.70 \\
\hline $\mathrm{C}^{\prime} \ldots \ldots \ldots \ldots \ldots \ldots \ldots$ & 1.96 & 2.7 & 9.5 & 0.9 & 3.8 & 0.06 & -1.40 & -0.45 & -0.57 \\
\hline D3 & 1.49 & -7.2 & -0.9 & 1.3 & 1.3 & -0.03 & -1.60 & -0.44 & -0.55 \\
\hline
\end{tabular}

\footnotetext{
${ }^{\text {a }}$ Distance relative to nucleus B1.

${ }^{\mathrm{b}}$ Flux measured $\left(\times 10^{-14} \mathrm{ergs} \mathrm{s}^{-1} \mathrm{~cm}^{-2}\right)$ using a circular aperture of 0.9 . Uncertainties $10 \%-15 \%(\sim 25 \%$ for B2).

${ }^{c}$ Internal extinction derived from the $\mathrm{H} \alpha / \mathrm{H} \beta$ ratio. Error in $A_{V}$ is 0.5 mag $\left(0.6\right.$ for $\mathrm{B} 1$ and $\mathrm{C}^{\prime}$, and $\sim 0.7$ for $\left.\mathrm{B} 2\right)$.

${ }^{\mathrm{d}}$ Average error \pm 0.13 .

e Average error \pm 0.05 .

${ }^{\mathrm{f}}$ Average error \pm 0.05 . In all cases, emission-line ratios are derived using extinction-corrected flux values.
} 

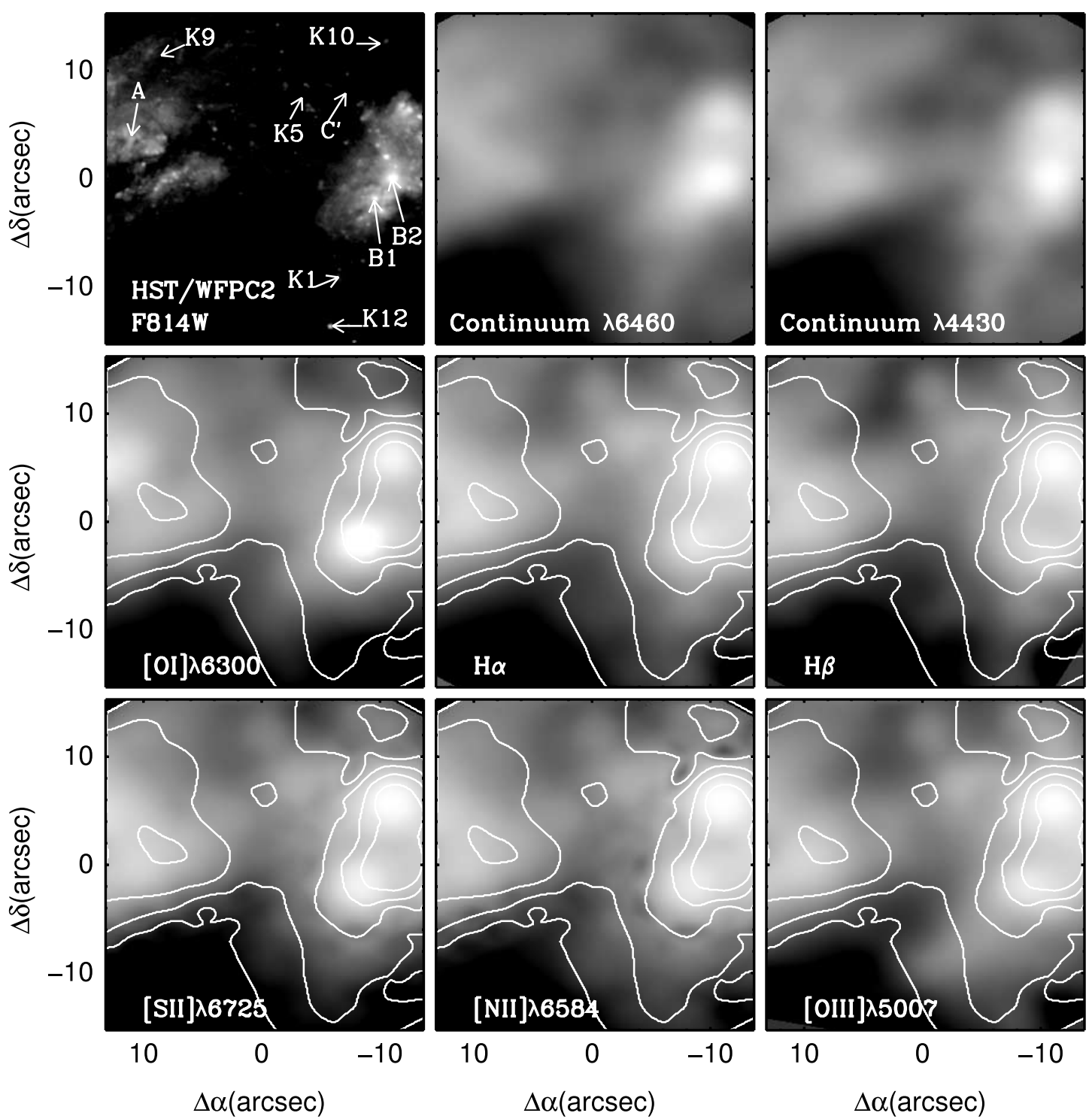

$\Delta \alpha(\operatorname{arcsec})$

FIG. 3. - Emission-line free stellar continuum and emission-line images for Arp 299 obtained with INTEGRAL SB3 bundle. NGC 3690 (B1 and B2) is located to the west and IC 694 (A) to the east. The contours represent the red continuum at $\lambda 6460$. The HST WFPC2 F814W image is shown for comparison, where several regions of interest discussed in the text are marked. All the images are shown on a logarithmic scale, and the color code represents different intensity levels in each of the individual maps. North up, and east is to the left. [See the electronic edition of the Journal for a color version of this figure.]

diameter 2 ".7, scales of $\simeq 0.5 \mathrm{kpc}$ ). In spite of that, the interface region between the two galaxies, and the external regions, show well-defined features such as the bridge to the south of region $\mathrm{K} 5$ connecting NGC 3690 and IC 694 and the elongated emission connecting $\mathrm{K} 1$ toward $\mathrm{K} 12$ source as part of a tail-like structure located to the southwest of NGC 3690 (see Fig. 1).

The general structure of the low surface brightness ionized gas in the interface region, as traced by for example $\mathrm{H} \alpha$ (Fig. 3), is similar to that of the stellar component. However, several differences exist. Diffuse ionized gas is detected northeast of NGC 3690 , associated with the $\mathrm{K} 5$ and $\mathrm{C}^{\prime}$ knots; the latter region shows $\mathrm{Pa} \alpha$ emission and SN activity in radio (Neff et al. 2004), and is part of a bridge of ionized material (see Fig. 3, $\mathrm{H} \alpha$ map) to the north of the stellar bridge that connects the two individual galaxies. The [O III] $\lambda 5007$ emission map presents an elongated structure located toward the southeast of B1 and B2 that traces an area where the ionized gas is in a high-excitation state ( $\operatorname{see} \oint 6.1$ ). However, as shown in the next two sections, the most important morphological variations are associated with the main bodies of the galaxies when observed with the higher angular resolution SB2 bundle.

\subsection{IC 694}

Previous studies have concluded that IC 694 is a spiral galaxy that, due to the merging process, shows a very disrupted morphology (e.g., Gehrz et al. 1983; Stanford \& Wood 1989; Hibbard \& Yun 1999). The stellar structure as seen from both the HST and the INTEGRAL continuum images (see Fig. 4) is divided into two main substructures: (1) The arm (region centered around A7) of about $1.8 \mathrm{kpc}$ in length, that could be one of the original spiral arms disrupted and deformed as a consequence of the merging process, and (2) the main body of the galaxy, formed by the dustenshrouded infrared nucleus A (AAH00), and surrounding regions. The two structures are well separated (distances of $\simeq 1 \mathrm{kpc}$ ) by what seems to be a dust lane, which is clearly visible in the HST near-IR color maps (AAH00). The effect of the internal extinction is remarkable in the continuum images as the relative surface brightness of the regions, in particular nucleus $\mathrm{A}$ 

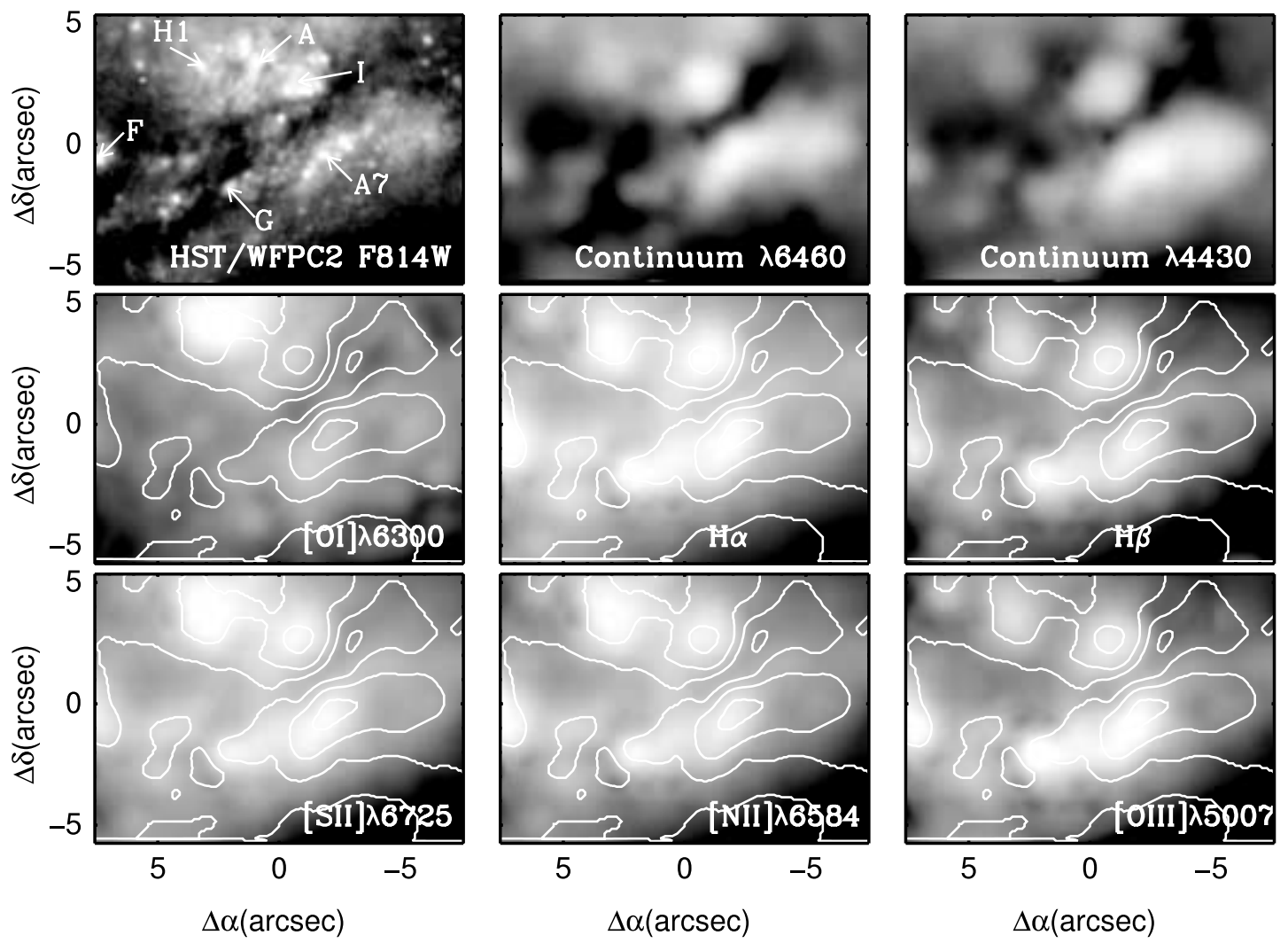

FIG. 4. - Emission-line free stellar continuum and emission-line images for IC 694 obtained with INTEGRAL SB2 bundle. The contours represent the red continuum at $\lambda 6460$. The HST WFPC2 F814W image is also shown for comparison, with several regions of interest marked on it. All the images are shown on a logarithmic scale, and the color code represents different intensity levels in each of the individual maps. North up, and east is to the left. [See the electronic edition of the Journal for a color version of this figure.]

and surrounding regions, changes with wavelength (see Fig. 4 and discussion in $\S 5.2$ ).

The ionized gas distribution (see Fig. 4) shows several distinct features worth mentioning. The nucleus of the galaxy (A) appears as a weak optical line emitting source, whereas the regions of high surface brightness are located at different positions (e.g., part of the arm and toward east, and regions close to A) and separated up to $1 \mathrm{kpc}$ from the nucleus. However, in the near-IR A is the brightest $\mathrm{Pa} \alpha$ and $\mathrm{Br} \gamma$ source (see AAH00; Sugai et al. 1999; see also $\S 5.2$ ), indicating that the extinction toward the nucleus is very high (using $\mathrm{Pa} \alpha / \mathrm{H} \alpha$ ratio, $A_{V}=6.1 \pm 0.7$ ). There are regions (e.g., knot $\mathrm{G}$ ) that appear as a faint continuum sources, but that are clearly identified as strong optical line emitters. In addition to differences in the morphology of the ionized gas (i.e., excitation conditions) with respect to that of the continuum, there are also differences in the ionized gas distribution as traced by several lines. The hydrogen recombination lines and the [N II] $\lambda 6584$ and [S II] $\lambda \lambda 6717,6731$ emission lines show a similar distribution, dominated by the emission from regions located on the arm and toward the east, and in the regions surrounding the nucleus. However, the shock tracer [O I] 26300 line is concentrated in region $\mathrm{H} 1$, while the higher excitation line [O III] 25007 appears to be brightest in regions A7 and G. These differences in the distribution of the emission-line gas are not only due to internal extinction effects, but they also reflect changes in the ionization conditions as a function of location within the galaxy (see $\S 6.1$ ).

\subsection{NGC 3690}

The stellar light distribution as observed with the HST shows an irregular morphology dominated by the emission coming from the high surface brightness region B2 (identified therefore as the optical nucleus), and from region $\mathrm{B} 1$ and complex $\mathrm{C}^{9}$ located at distances of 0.49 and $1.3 \mathrm{kpc}$ from B2, respectively (see Fig. 5). However, at near- and mid-IR wavelengths the true nucleus of the galaxy (see discussion in $\S 10.2$ ), B1, becomes the brightest continuum region in NGC 3690 (see AAH00; Satyapal et al. 1999). This disordered morphology could be the combination of the original spiral structure with young star-forming regions such as the complex $\mathrm{C}$, and regions $\mathrm{D} 3$ or $\mathrm{C}^{\prime}$, newly formed as a consequence of the interaction process as suggested by several authors (e.g., Gehrz et al. 1983; Stanford \& Wood 1989; Baan \& Haschick 1990; Hibbard \& Yun 1999).

The distribution of the ionized gas (Fig. 5) is simpler than that of IC 694. The optical nucleus (B2) appears as an extremely faint line emitter, and the structure of the ionized gas is dominated by two high surface brightness regions separated by $1.8 \mathrm{kpc}$, nucleus $\mathrm{B} 1$, and complex $\mathrm{C}$ (with the peak located toward $\mathrm{C} 2$ ), as previously found with near-IR hydrogen recombination lines (Sugai et al. 1999; AAH00). The brightest emission-line peak is associated with $\mathrm{C} 2$ for all lines but the shock tracer $\left[\mathrm{O}_{\mathrm{I}}\right] \lambda 6300$. For the latter, nucleus B1 is the brightest emitter, as also observed with the near-IR [Fe II] $\lambda 1.64 \mu \mathrm{m}$ line (AAH00). Analyzing in detail the secondary emission-line peaks associated with B1 and surrounding areas, it is interesting to note that the high-excitation [O III] $\lambda 5007$ emission is dominated by $\mathrm{B} 1$, while the $\mathrm{H} \alpha$ and $\mathrm{H} \beta$ emission peaks are associated with region B16 (which is located

\footnotetext{
9 As explained in footnote 7 , we will use the term complex $\mathrm{C}$ introduced by Gehrz et al. (1983) for the regions C1, C2, and C3 (see Fig. 1).
} 

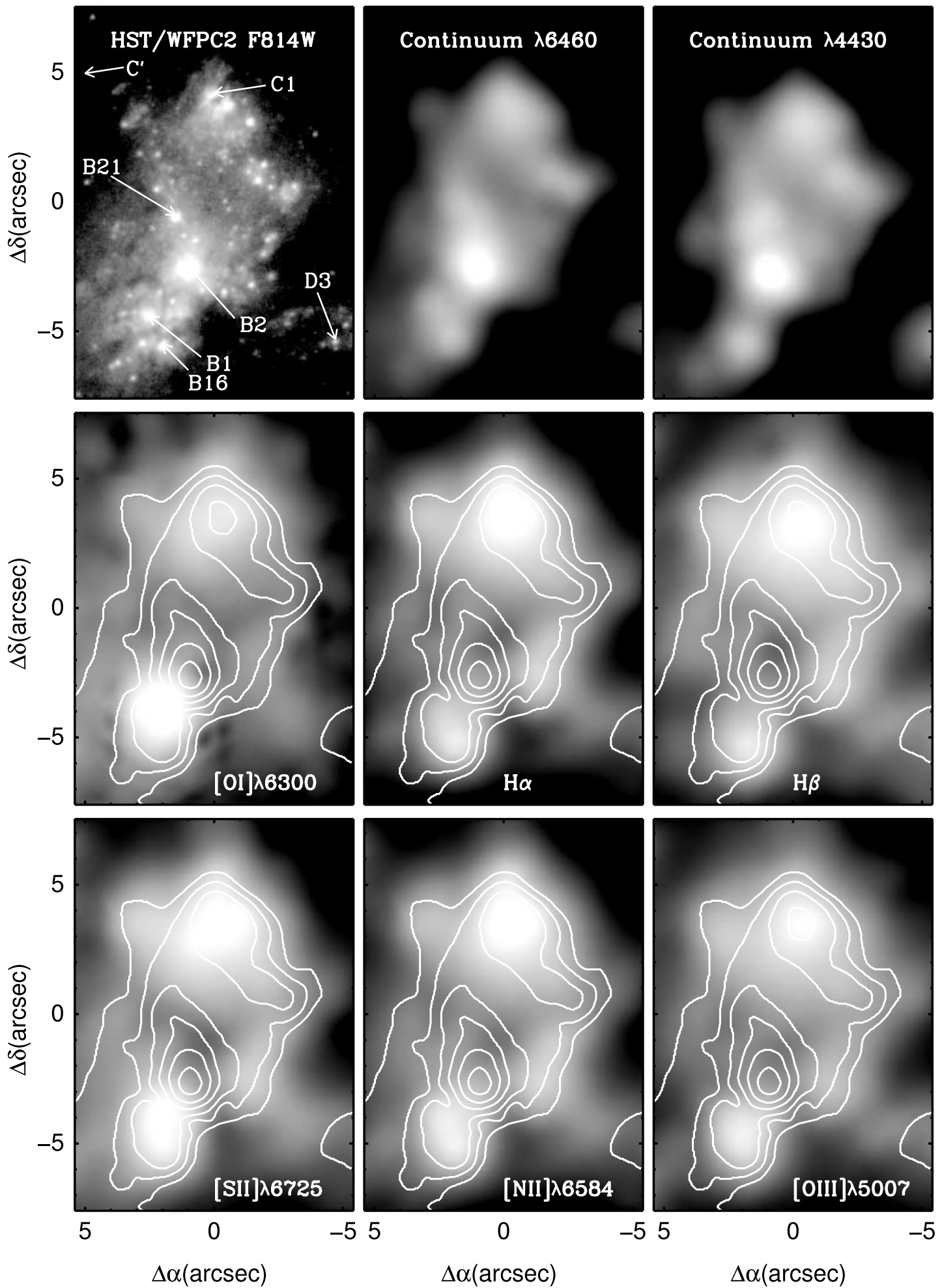

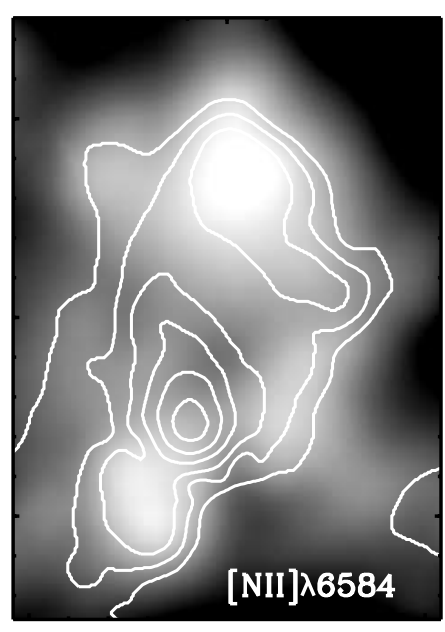

5

0

$\Delta \alpha(\operatorname{arcsec})$

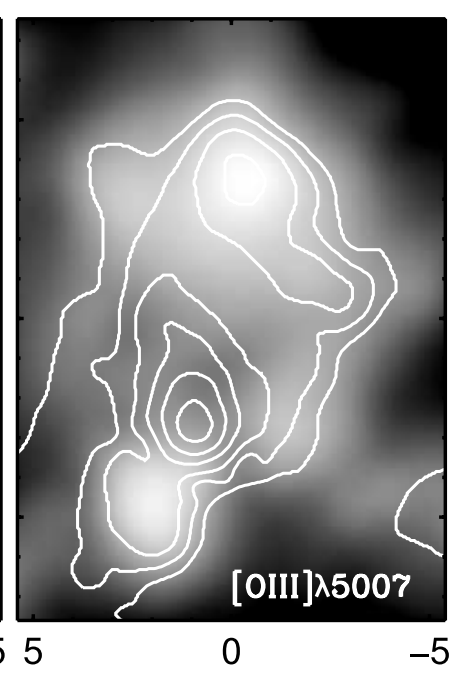

$\Delta \alpha(\operatorname{arcsec})$

FIG. 5.-Emission-line free stellar continuum and emission-line images for NGC 3690 obtained with INTEGRAL SB2 bundle. The contours represent the red continuum at

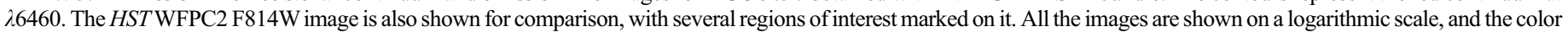

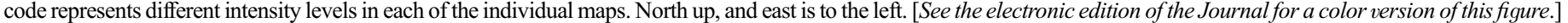

$0.26 \mathrm{kpc}$ southwest of $\mathrm{B} 1$, and identified in the $\lambda 4475$ continuum map). Low-excitation collisional lines [N $\mathrm{N}$ ] $\lambda 6584$ and [S II] $\lambda \lambda 6717,6731$ appear to have secondary peaks in both B1 and B16. These changes in the emission-line distribution represent changes in the ionization conditions, and most likely in the ionizing source (AGNs, young stellar clusters, and shocks) on scales of less than $0.3 \mathrm{kpc}$.

\section{INTERNAL DUST DISTRIBUTION AND EXTINCTION EFFECTS}

As mentioned in the previous section, part of the complex structure observed in the continuum and emission-line light distributions is due to large amounts of dust producing spatially dependent internal extinction effects. The two-dimensional extinction 

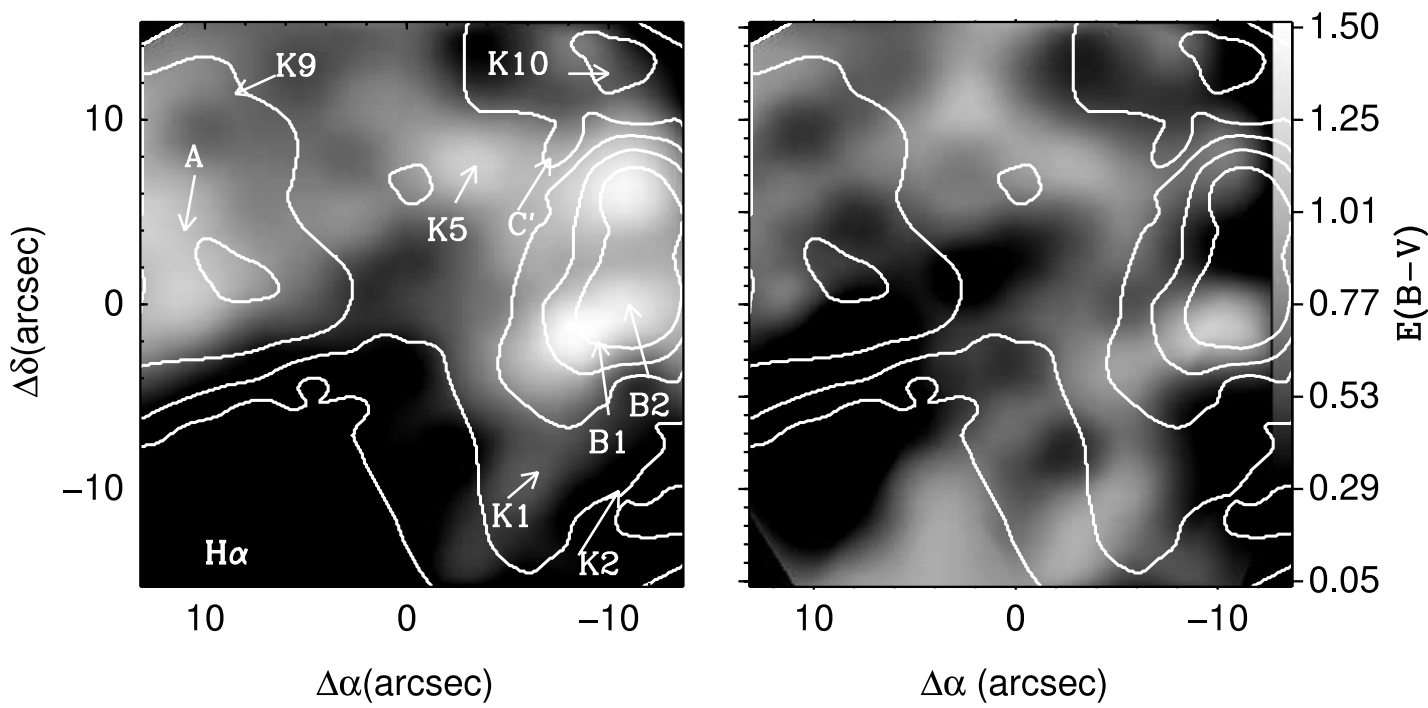

FIG. 6. - Left: Extinction-corrected $\mathrm{H} \alpha$ map of the Arp 299 system (including the interface region), shown on a logarithmic scale. Right: Extinction map of the interface region obtained from the line ratio $\mathrm{H} \alpha / \mathrm{H} \beta$, shown on a linear scale. Contours are as in Fig. 3. [See the electronic edition of the Journal for a color version of this figure.]

maps of the gas (Figs. 6, 7, and 8) have been derived using the $\mathrm{H} \alpha / \mathrm{H} \beta$ line ratio, a foreground dust screen model, and a mean interstellar extinction law based on that of Savage \& Mathis (1979). No correction for the presence of underlying stellar hydrogen absorption lines has been applied. Assuming an equivalent width value of $\mathrm{EW}_{\mathrm{abs}}(\mathrm{H} \alpha)=\mathrm{EW}_{\mathrm{abs}}(\mathrm{H} \beta) \sim 2 \AA$, the derived extinction could be overestimated by as much as $\Delta E(B-V) \sim 0.6$ for regions with extremely faint $\mathrm{H} \beta$ emission [e.g., $\mathrm{EW}(\mathrm{H} \beta) \leq$ $20 \AA]$. The values for the visual extinction $\left[A_{V}=3.1 \times E(B-\right.$
$V)]$ in particular regions are given in Tables 1,2 , and 3 . The results suggest that the highest extinguished regions are, in general, associated with the nuclei and some particular regions (such as $\mathrm{C}^{\prime}$ ), while the external $\mathrm{H}$ II regions are mostly less affected by the presence of dust.

\subsection{Interface Region}

The extinction in the whole area covered by the SB3 bundle on scales of about 3".0 (i.e., $0.6 \mathrm{kpc}$ ), is relatively uniform (see
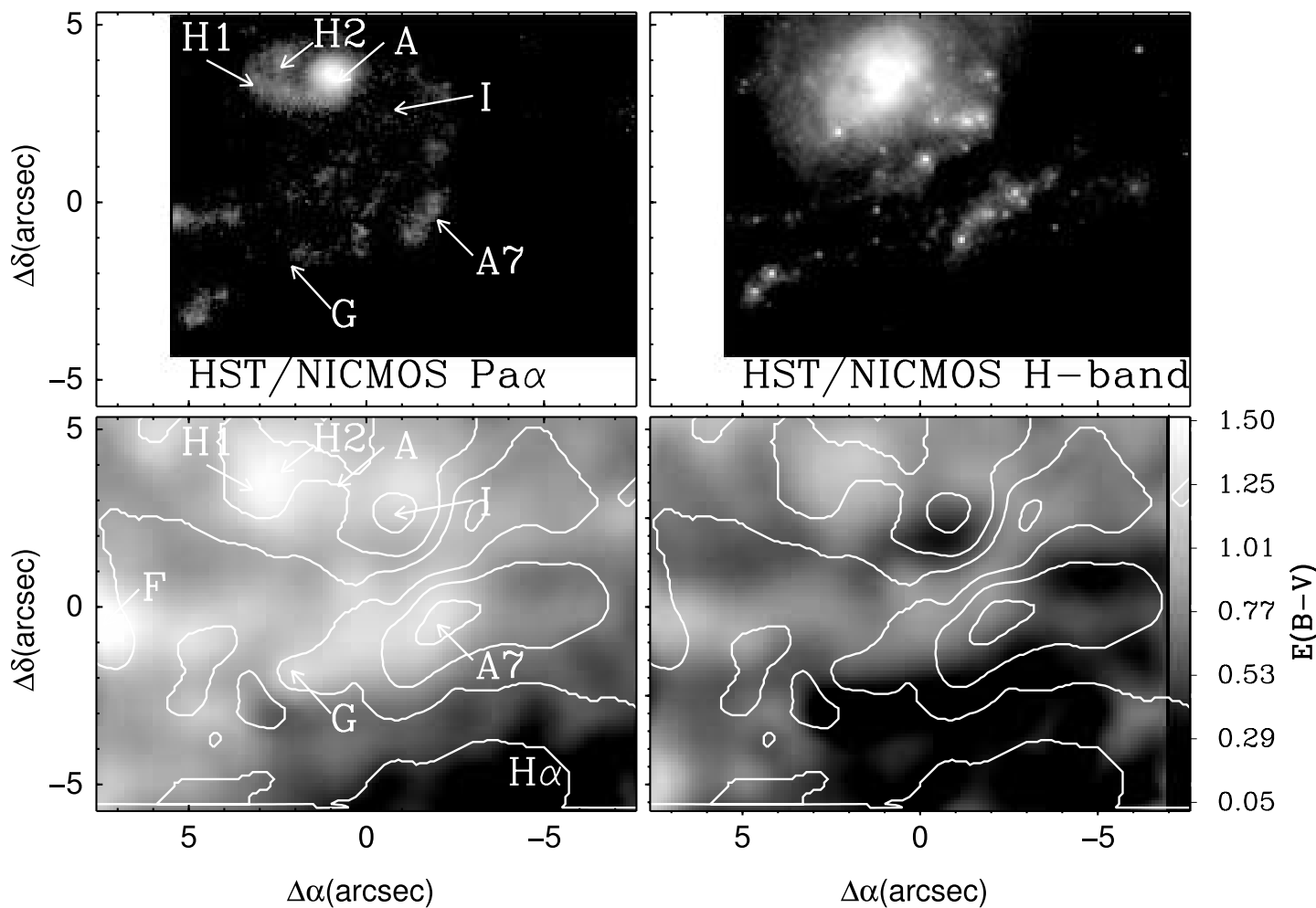

FIG. 7.-IC 694. Top left: HST NICMOS continuum-subtracted Pa $\alpha$ emission (F190N - F187N). Top right: HST NICMOS H-band continuum. Bottom left: INTEGRAL $\mathrm{H} \alpha$ extinction-corrected map. Bottom right: Extinction map obtained with $\mathrm{H} \alpha / \mathrm{H} \beta$ line ratio. As a reference several regions in the two fields are marked, and the red continuum contours are superimposed on the interpolated maps. The NICMOS images are shown covering the same FOV as the INTEGRAL data. All the images are shown on a logarithmic scale except for the extinction map, where a linear scale has been applied. [See the electronic edition of the Journal for a color version of this figure.] 

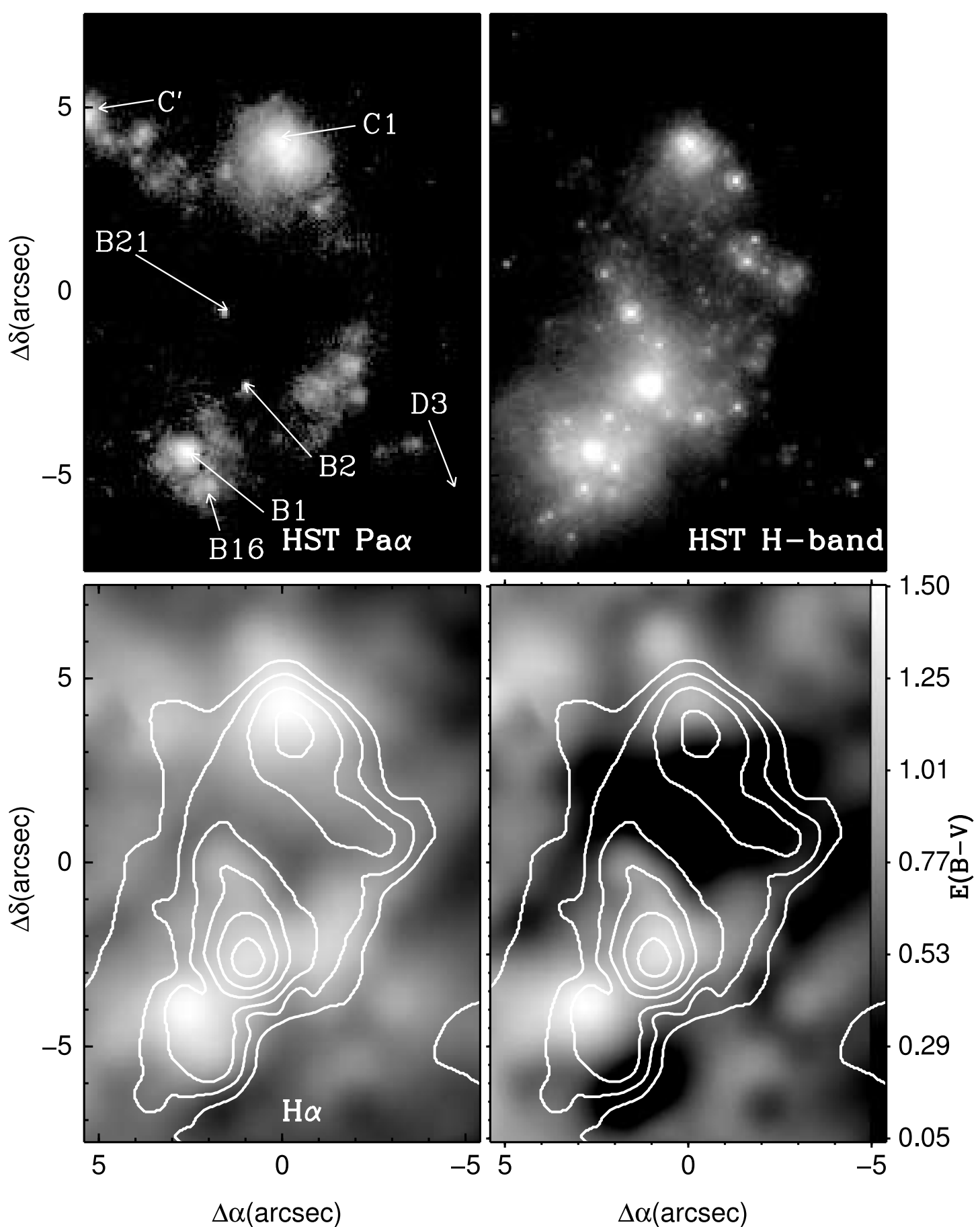

Fig. 8.-NGC 3690. Top left: HST NICMOS continuum-subtracted Pa $\alpha$ emission (F190N - F187N). Top right: HST NICMOS H-band continuum. Bottom left: INTEGRAL $\mathrm{H} \alpha$ extinction-corrected map. Bottom right: Extinction map obtained with $\mathrm{H} \alpha / \mathrm{H} \beta$ line ratio. As a reference several regions are marked, and the red continuum contours are superimposed on the interpolated maps. The NICMOS images are shown covering the same FOV as the INTEGRAL data. All the images are shown on a logarithmic scale except for the extinction map, where a linear scale has been applied. [See the electronic edition of the Journal for a color version of this figure.]

Fig. 6). It has an average value of $A_{V}=1.9$ mag on the selected individual regions, and ranges from $A_{V}(\mathrm{~K} 2)=0.9 \pm 0.7 \mathrm{mag}$ to $A_{V}(\mathrm{~K} 5)=2.9 \pm 0.7 \mathrm{mag}$ (see Table 1 for specific values on all the individual regions).

\subsection{IC 694}

On scales of about 1".0 (i.e., $0.21 \mathrm{kpc}$ ), the internal extinction in the visual covers a wide range of values from about $A_{V}=$ $0.6 \pm 0.5 \mathrm{mag}$ in the southwestern section of the galaxy to $A_{V}=$ $3.4 \pm 0.5$ mag in region $\mathrm{H}_{2}$, close to nucleus A [see Table 2 for specific values, and Fig. 7 for the two-dimensional $E(B-V)$ distribution]. As can be seen from Figure 7, the $\mathrm{H} \alpha$ extinctioncorrected light distribution shows an overall structure similar to that of the $\mathrm{Pa} \alpha$ line except for the region associated with the peak of the emission. While nucleus A, identified as the brightest nearinfrared source in the $H S T$ images (Fig. 7, see also AAH00), is also the dominant $\mathrm{Pa} \alpha$ line emitter, the peak of the extinctioncorrected $\mathrm{H} \alpha$ distribution is located in regions $\mathrm{H} 1$ and $\mathrm{H}_{2}, 0.3$ and $0.5 \mathrm{kpc}$ toward the east of the nucleus. Also, region F, located $1.58 \mathrm{kpc}$ to the southeast of A, appears as a strongly absorbed 
TABLE 4

Pa $\alpha / \mathrm{H} \alpha$-Based Internal Extinction Estimates

\begin{tabular}{|c|c|c|c|}
\hline Region & 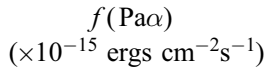 & $f(\mathrm{~Pa} \alpha) / f(\mathrm{H} \alpha)$ & $\begin{array}{c}A_{V} \\
(\mathrm{mag})\end{array}$ \\
\hline 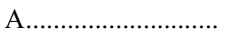 & 167 & 5.8 & 6.1 \\
\hline A1 .......................... & 2.74 & 0.1 & $\ldots$ \\
\hline A $2 \ldots \ldots \ldots \ldots \ldots \ldots \ldots \ldots$ & 5.68 & 0.2 & 0.9 \\
\hline A $3 \ldots \ldots \ldots \ldots \ldots \ldots \ldots$ & 4.37 & 0.2 & 0.9 \\
\hline A4 ........................... & 9.62 & 0.3 & 1.5 \\
\hline A $5 \ldots \ldots \ldots \ldots \ldots \ldots \ldots \ldots$ & 8.41 & 0.1 & $\ldots$ \\
\hline A6.......................... & 2.73 & 0.1 & $\ldots$ \\
\hline A7 .......................... & 10.5 & 0.2 & 0.9 \\
\hline $\mathrm{H} 1 \ldots \ldots \ldots \ldots \ldots \ldots \ldots \ldots$ & 12.6 & 0.2 & 0.9 \\
\hline $\mathrm{H} 2 \ldots \ldots \ldots \ldots \ldots \ldots \ldots \ldots \ldots$ & 17.3 & 0.4 & 2.0 \\
\hline I …........................... & 5.11 & 0.1 & $\ldots$ \\
\hline I1 …..................... & $\ldots$ & $\ldots$ & $\cdots$ \\
\hline B1 …...................... & 66.7 & 0.7 & 2.8 \\
\hline 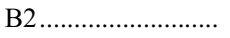 & 6.10 & 0.2 & 0.9 \\
\hline B11 ......................... & 9.77 & 0.2 & 0.9 \\
\hline B16 .......................... & 18.5 & 0.2 & 0.9 \\
\hline B21 ........................ & 4.14 & 0.1 & $\ldots$ \\
\hline 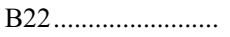 & 5.15 & 0.2 & 0.9 \\
\hline B25 ......................... & 11.0 & 0.2 & 0.9 \\
\hline $\mathrm{C} 1 \ldots \ldots \ldots \ldots \ldots \ldots \ldots$ & 85.3 & 0.3 & 1.5 \\
\hline $\mathrm{C} 2 \ldots \ldots \ldots \ldots \ldots \ldots \ldots \ldots$ & 76.3 & 0.2 & 0.9 \\
\hline 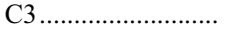 & 10.0 & 0.1 & $\cdots$ \\
\hline 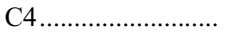 & 4.70 & 0.1 & $\ldots$ \\
\hline C6 ........................... & 9.16 & 0.2 & 0.9 \\
\hline $\mathrm{C}^{\prime} \ldots \ldots \ldots \ldots \ldots \ldots \ldots \ldots$ & 38.1 & 4.1 & 5.6 \\
\hline D3 & 0.15 & 0.01 & $\ldots$ \\
\hline 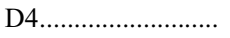 & 4.81 & 0.2 & 0.9 \\
\hline
\end{tabular}

Notes.-Fluxes of $\mathrm{Pa} \alpha$ measured over the selected regions using the HST ( $\mathrm{F} 190 \mathrm{~N}-\mathrm{F} 187 \mathrm{~N}$ ) image. Average uncertainty in $A_{V}$ is $0.7 \mathrm{mag}$. Some values are discarded due to the noise affecting $\mathrm{Pa} \alpha$ and $\mathrm{H} \alpha$ low-surface brightness.

region $\left(A_{V}=3.2 \pm 0.5 \mathrm{mag}\right)$, and as a strong $\mathrm{H} \alpha$ emitter after correcting for extinction. Additional secondary $\mathrm{H} \alpha$ sources are mostly associated with star-forming regions (the arm, a region toward the west of A) at distances of 1-1.6 kpc from the nucleus, and delineating what seems to be a spiral-like arm structure previously identified in the $\mathrm{Pa} \alpha$ emission-line map.

\subsection{NGC 3690}

This galaxy presents the widest range of extinction (derived from the $\mathrm{H} \alpha / \mathrm{H} \beta$ ratio) for the entire Arp 299 system with values that for the individual regions range from $A_{V}(\mathrm{C} 4)=0.5 \pm 0.5 \mathrm{mag}$ to $A_{V}$ (B2) $=3.9 \pm 0.6 \mathrm{mag}$ (see Table 3). Also, nucleus B1 in this galaxy is the region of the Arp 299 system that has the highest associated $\mathrm{H} \alpha / \mathrm{H} \beta$-based extinction, $A_{V} \simeq 4.6$ mag (Fig. 8).

The overall structure of the extinction-corrected $\mathrm{H} \alpha$ line distribution agrees well with that of the Pa $\alpha$ line (Fig. 8; see also AAH00) not only on the high surface brightness regions, such as the nuclei $\mathrm{B} 1$ and $\mathrm{C} 1$, but also on the low surface brightness region, including B2 identified as the optical nucleus.

\subsection{Extinction Estimate Using Optical and Near-IR Emission Lines}

Additional line ratios involving IR hydrogen recombination lines (e.g., $\mathrm{Pa} \alpha / \mathrm{H} \alpha, \mathrm{Br} \gamma / \mathrm{Pa} \alpha$, and $\mathrm{Pa} \alpha / \mathrm{Pa} \beta$ ), can provide better estimates of the internal extinction in highly extinguished regions as those identified in Arp 299 (see Table 4).

The Pa $\alpha$ image of the individual galaxies (see Figs. 7, 8, and AAH00) has been used for measuring all the regions under study with apertures of 1 ." 0 for IC 694 and of 0 ." 9 for NGC 3690 to match our ground-based apertures; the INTEGRAL $\mathrm{H} \alpha$ fluxes are given in Tables 2 and 3. In most regions extinction estimates based on only optical and optical + infrared lines agree within the uncertainties (Table 4). On the other hand, there are two regions, the nucleus of IC 694 (A) and $\mathrm{C}^{\prime}$, where the use of the optical + infrared lines indicates a larger extinction than using only the optical lines, i.e., $A_{V}(\mathrm{~Pa} \alpha / \mathrm{H} \alpha) \simeq 2 \times A_{V}(\mathrm{H} \alpha / \mathrm{H} \beta)$. This means that the extinctions derived using the $\mathrm{H} \alpha / \mathrm{H} \beta$ ratio are in general consistent with the ones derived using the $\mathrm{Pa} \alpha / \mathrm{H} \alpha$ ratio, except in highly absorbed regions, where there is an underestimate when only the optical lines are used. These extinctions are similar to those measured in the M51 nuclear $\mathrm{H}$ II regions (Calzetti et al. 2005) and in other luminous infrared galaxies (LIRGs; Alonso-Herrero et al. 2006) using the same near-IR/ optical emission lines.

\section{TWO-DIMENSIONAL IONIZATION STRUCTURE}

The ionization level for the individual regions identified in Arp 299 has been derived using standard optical emission line diagnostic diagrams (BPT diagrams; Baldwin et al. 1981; Veilleux \& Osterbrock 1987). H II-like ionization (i.e., star formation) is present in the majority of regions of the individual galaxies, except for nucleus B1, which is clearly in a Seyfert-like state (i.e., there is an AGN present). LINER-like ionization is mainly present on the interface (Fig. 9; see also Tables 1, 2, and 3 for specific values of the emission-line ratios). Several regions show changes in the classification depending on the specific diagram used; these
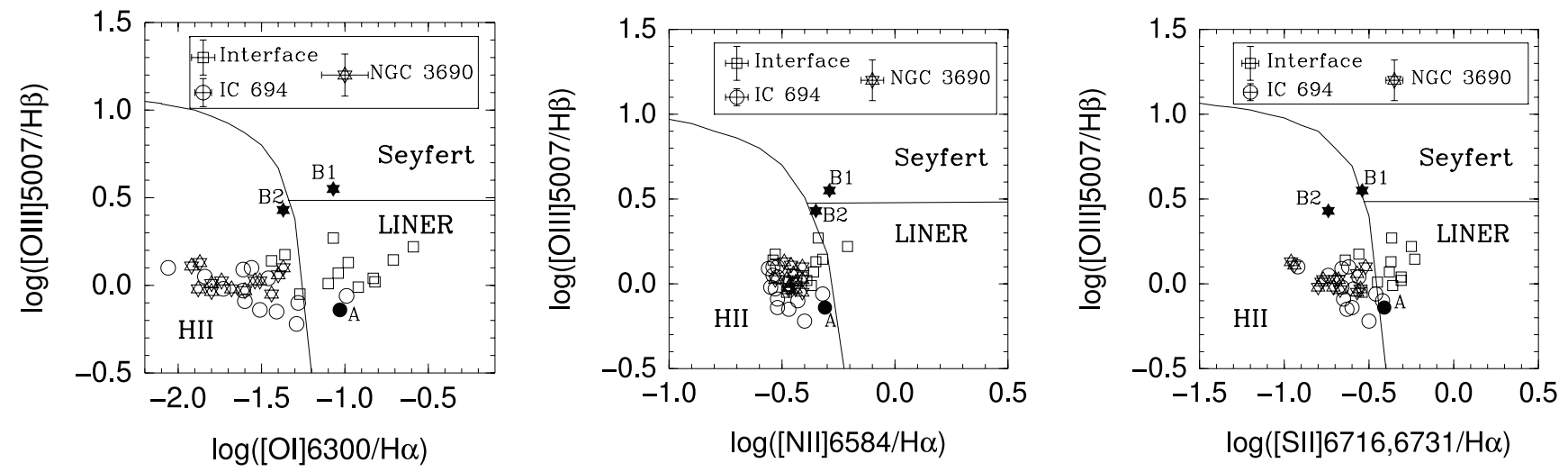

FIG. 9.-Optical emission line diagnostic diagrams for the studied regions of Arp 299. Filled symbols represent nuclei A and B1, and region B2. Note that B1, as observed with the SB2 bundle, is classified as Seyfert galaxy according to these diagrams. The open symbols are the regions given in Tables 1, 2, and 3. Typical uncertainties in the line ratios of each individual galaxy are shown. [See the electronic edition of the Journal for a color version of this figure.] 
variations have been interpreted as a consequence of changes in the dominant ionizing mechanism from photoionization to shocks (e.g., Monreal-Ibero et al. 2006 and references therein). In particular, the $\left[\mathrm{O}_{\mathrm{III}}\right] \lambda 5007 / \mathrm{H} \beta$ to $\left[\mathrm{O}_{\mathrm{I}}\right] \lambda 6300 / \mathrm{H} \alpha$ line ratio is more sensitive to, and therefore a more reliable tracer of, changes in the ionization conditions due to fast shocks than any of the other optical emission line ratios involving [N II] $\lambda 6584 / \mathrm{H} \alpha$ or [S II] $\lambda 6725 / \mathrm{H} \alpha$ ratios (Dopita \& Sutherland 1995). In the following subsections a more detailed discussion of the two-dimensional ionization structure of the ionized gas distribution based on the [O III] $\lambda 5007 / \mathrm{H} \beta-[\mathrm{O}$ I] $\lambda 6300 / \mathrm{H} \alpha$ diagram and on the [O III] $\lambda 5007 / \mathrm{H} \beta$, and $\left[\mathrm{O}_{\mathrm{I}}\right] \lambda 6300 / \mathrm{H} \alpha$ maps for the interface, IC 694 , and NGC 3690 is given.

\subsection{Interface Region: Evidence for an Off-Nuclear Seyfert-like Ionization Cone}

The $[\mathrm{O}$ III $] \lambda 5007 / \mathrm{H} \beta-\left[\mathrm{O}_{\mathrm{I}}\right] \lambda 6300 / \mathrm{H} \alpha$ line ratios have been obtained for all the INTEGRAL/SB3 individual spectra (Fig. 10). Although these data include the high surface brightness nuclear regions of IC 694 and NGC 3690, and their external regions, they also cover the area that has been identified as the interface zone, i.e., the extended low surface brightness region connecting the two galaxies up to distances of about $4 \mathrm{kpc}$ from their nuclei. Changes in the ionization structure traced by the $[\mathrm{O}$ III $] \lambda 5007 / \mathrm{H} \beta$ and $\left[\mathrm{O}_{\mathrm{I}}\right] \lambda 6300 / \mathrm{H} \alpha$ two-dimensional spatial distribution line ratios are also shown (Fig. 10).

Three different types of ionization, H II, LINER, and Seyfertlike, are identified, and the line ratio maps clearly show that the ionization structure has a well-defined spatial distribution. The majority of the regions associated with the two individual galaxies (marked with two isocontours in the figure) are dominated by $\mathrm{H}$ II-like ionization, as expected from the intense star formation taking place there (see more details in $\S 9$ ). There are some exceptions; nucleus B1 shows LINER-like activity at this angular resolution. The Seyfert-like ionization is clearly resolved with a size of about $7^{\prime \prime}(\sim 1.5 \mathrm{kpc})$ and has a conical morphology, with an opening angle of about $54^{\circ}$ and its apex located in nucleus B1 (at a projected distance of about $1.5 \mathrm{kpc}$ from the peak of the nebula) within the angular resolution (see Fig. 10). Moreover, this highly ionized gas is not associated with any particular concentration of stellar mass according to the optical (WFPC2/ F814W; see Fig. 1) HST continuum image. Off-nuclear Seyfertlike nebulae at distances of few kiloparsecs from the nucleus have already been reported in other (ultraluminous) LIRGs with Seyfert nuclei (Mrk 273: Colina et al. 1999) and have been interpreted as photoionization of extranuclear interstellar gas by the AGN. Therefore, our data strongly suggest photoionization by a radiation cone escaping from a central dust-enshrouded AGN source located in B1. The highly ionized conical structure in Arp 299 (NGC 3690) is also detected at distances of up to $4 \mathrm{kpc}$ from B1; however, the ionization associated with these outer regions of the cone is LINER-like rather than Seyfert-like (Fig. 10). Therefore, it seems that within the cone there are two ionization regimes, which are well separated spatially. At projected distances from B1 smaller than about $2 \mathrm{kpc}$ the ionization is likely due to radiation coming from the AGN located in $\mathrm{B} 1$, while at larger distances LINER-like ionization is dominant. This LINER-like ionization is also found in other areas of the interface region, but with a lower degree of excitation, as traced by the [O I ] $\lambda 6300 / \mathrm{H} \alpha$ ratio measured in these regions.

\subsection{IC 694}

Over the area covered by the INTEGRAL SB2 bundle, H II and LINER-like ionization are present in this galaxy (Fig. 11).
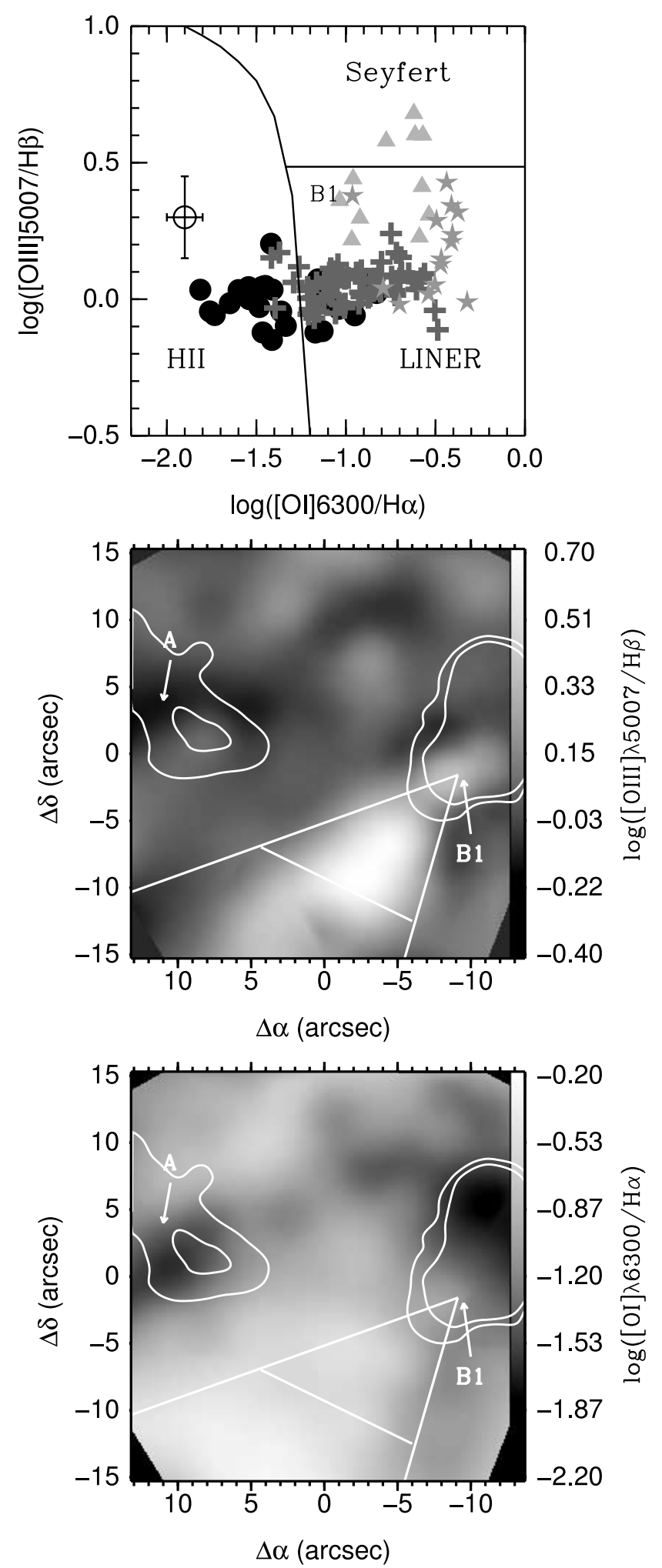

FIG. 10.-Diagnostic diagram and line ratio maps derived from INTEGRAL / SB3 data. Top: Diagnostic diagram with all the data from the SB3 bundle displayed. The symbol code is as follows: triangles represent values within the ionization cone, and at a distance of less than $2 \mathrm{kpc}$ from B1. Stars represent the zone within the cone at distances of 2-4 kpc from B1. Crosses mark the rest of the regions in the interface. Filled circles represent regions in IC 694 and NGC 3690 (defined as the regions within the isocontours). B1 has been included in the ionization cone, and labeled as a triangle. Middle: [O III] $25007 / \mathrm{H} \beta$ map. Nuclei A and B1 are marked. The white lines indicate the ionization cone. The transversal line to the axis of the cone separates the inner (with Seyfert-like regions) and outer (LINER-like) sections of the cone. Bottom: [O I] $\lambda 6300 / \mathrm{H} \alpha$ map. The labels and overlays are as in the middle panel. [See the electronic edition of the Journal for a color version of this figure.] 

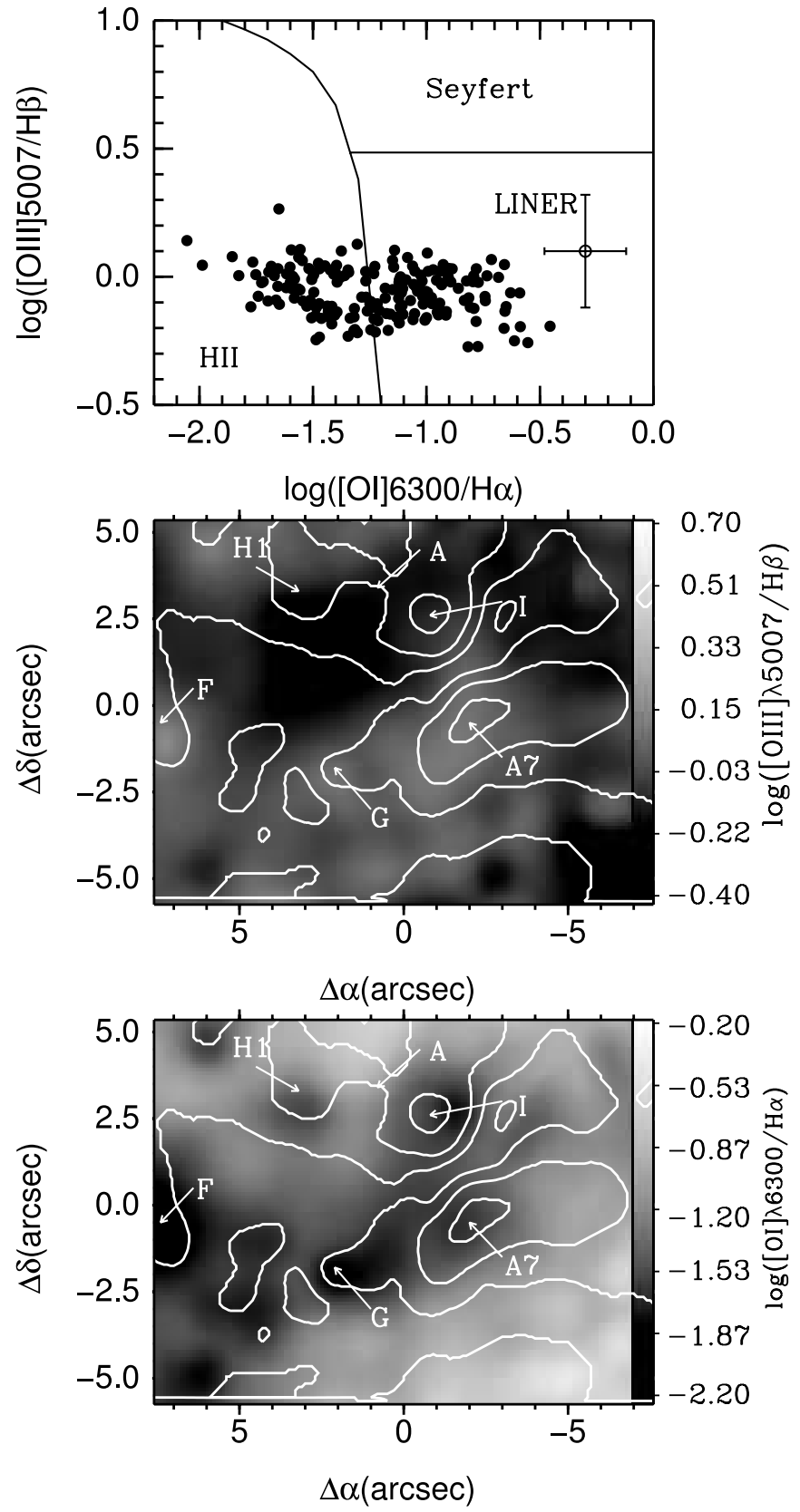

FIG. 11.-Emission-line diagnostic diagram and excitation maps based on line ratios for IC 694. Top: Diagnostic diagram obtained for the individual SB2 spectra. Middle and bottom: Diagnostic maps derived from reconstructed emissionline maps. As a reference we have marked several regions and superimposed red continuum contours. [See the electronic edition of the Journal for a color version of this figure.]

The main body of the galaxy, including the regions close to nucleus $A$ and the spiral arm structure $(A 7, G)$ toward the east $(F)$, is dominated by the star-forming knots. LINER-like ionization is present elsewhere, being more prominent in diffuse low surface brightness regions located at a projected distance of about $1.8 \mathrm{kpc}$. The $[\mathrm{N}$ II $] \lambda 6584 / \mathrm{H} \alpha$ and the $[\mathrm{S}$ II $] \lambda 6725 / \mathrm{H} \alpha$ line ratio maps (not shown) also have a similar structure, although, as explained above, they tend to indicate a lower ionization.

\subsection{NGC 3690}

Three types of excitation level (H II-, LINER-, and Seyfertlike) are present in this individual galaxy, as shown in the [O III $] \lambda 5007 / \mathrm{H} \beta-\left[\mathrm{O}_{\mathrm{I}}\right] \lambda 6300 / \mathrm{H} \alpha$ diagnostic diagram and in the
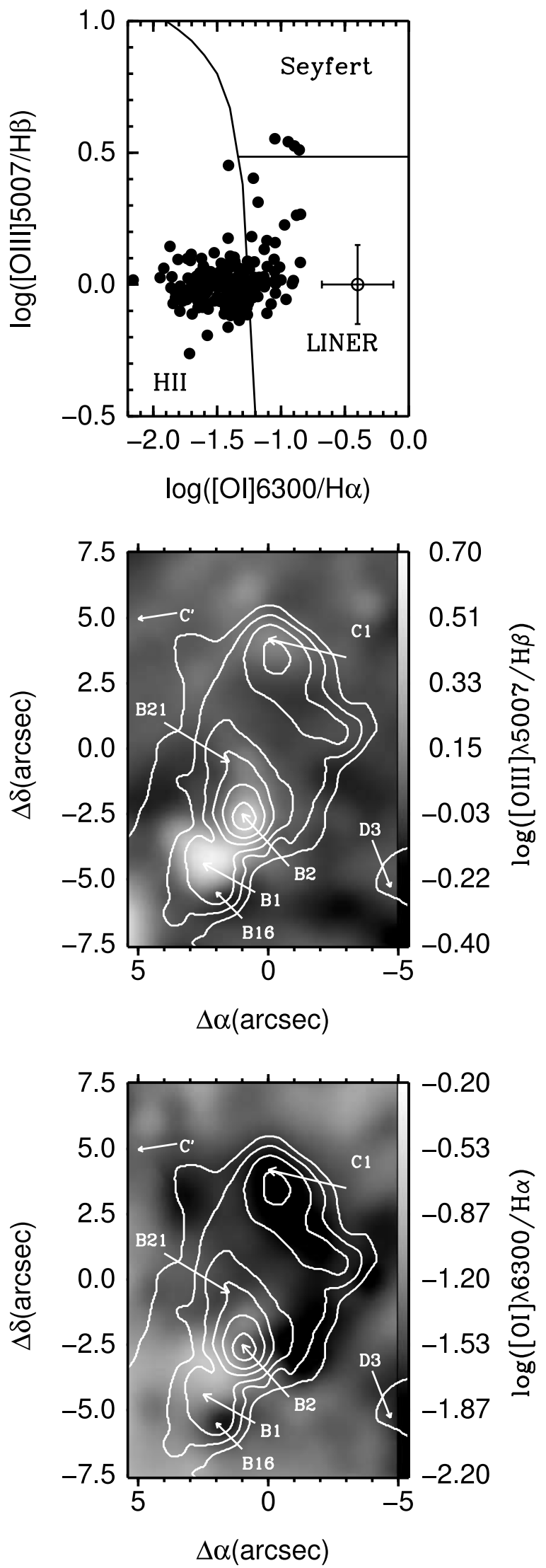

FIG. 12.-Emission-line diagnostic diagram and excitation maps based on line ratios for NGC 3690. Top: Diagnostic diagram obtained from the individual SB2 spectra. Middle and bottom: Diagnostic maps derived from reconstructed emissionline maps. As a reference we have marked several regions and superimposed red continuum contours. [See the electronic edition of the Journal for a color version of this figure.] 

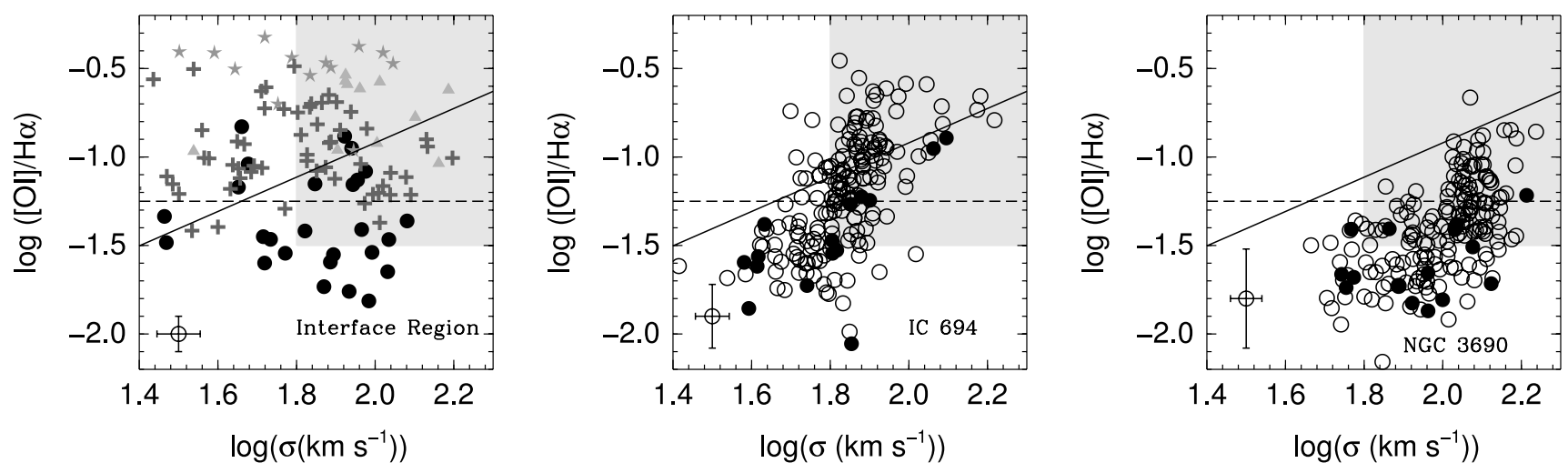

FIG. 13. - Relation between velocity dispersion and [O I] $\lambda 6300 / \mathrm{H} \alpha$ ratio for the three INTEGRAL pointings, i.e., interface region (left), IC 694 (middle), and NGC 3690 (right). In all cases the relation derived by Monreal-Ibero et al. (2006) for a set of ULIRGs is shown (solid line). The shaded area indicate the region covered by extranuclear regions of ULIRGs (Monreal-Ibero et al. 2006). The horizontal dashed line marks the average values separating H II and LINER-like ionization. Left: The symbol code is as in Fig. 10. Middle and right: Filled symbols indicate the fibers of the studied individual regions listed in Tables 2 and 3. [See the electronic edition of the Journal for a color version of this figure.]

two-dimensional maps (Fig. 12). Regarding nucleus B1, these higher resolution data give a clear Seyfert-like classification in all these line ratios (Fig. 9), and show that there is an extended zone of about $2^{\prime \prime}(0.4 \mathrm{kpc})$ in length associated with B1 with Seyfert characteristics. This is the inner section of the AGN-like ionization cone detected with the SB3 bundle, with its apex coincident with B1. Moreover, region B2 also presents a high-ionization state at the borderline between Seyfert galaxies and LINERs, a characteristic that could indicate the presence of a counterionization cone. The detection of Seyfert-like activity based on optical emission lines represent the first evidence in the optical for the presence of an AGN in B1 and supports the recent claim of an AGN based on X-ray (Della Ceca et al. 2002) and mid-IR (Gallais et al. 2004) data. Previous spectroscopic studies in the optical (Gehrz et al. 1983; Coziol et al. 1998; Keel 1984) did not find AGN emission, probably due to the large size of the slits (3". 6,2 ".5, and 8.0$)$. This is in agreement with our results, where the Seyfert nature of the B1 nucleus has been diluted in the SB3 $(\phi=2$ ".7) spectra, whereas in the higher spatial resolution SB2 $(\phi=0.9)$ spectra the classification is clear.

$\mathrm{H}$ II-like ionization dominates the rest of the main body of the galaxy (e.g., complex C), while LINER-like ionization is present in a few outer regions. The [O I] $\lambda 6300 / \mathrm{H} \alpha$ map has its peak displaced about 0 ! $7(0.14 \mathrm{kpc})$ to the north with respect to B1. Similar displacements are observed in the [N II] $26584 / \mathrm{H} \alpha$ and in the [S II] $\lambda 6725 / \mathrm{H} \alpha$ maps. These lines, and in particular [O I] $\lambda 6300 / \mathrm{H} \alpha$, are good tracers of shocks, and thus these shifts may indicate different ionization mechanisms around B1 on scales of $200 \mathrm{pc}$. These small-scale ionization changes need further investigation with high angular resolution spectroscopy.

\section{ELECTRON DENSITY}

Two-dimensional electron density maps of the ionized gas distribution have been derived for the entire Arp 299 system using the standard [S II] $\lambda 6716 /[\mathrm{S}$ II] $\lambda 6731$ lines ratio (Aller 1984; Osterbrock 1989). The average line ratios for the interface and the individual galaxies show a similar value, about $1.3 \pm 0.1$, indicating an average uniform electron density $\left(\sim 100 \mathrm{~cm}^{-3}\right)$ over the entire Arp 299 system. However, the ionization cone detected in NGC 3690 presents a well-defined gradient of this line ratio value, which roughly ranges from $1.2 \pm 0.15$ in the higher excitation (i.e., Seyfert-like) center of the cone to $1.8 \pm 0.15$ in the lower excitation (i.e., LINER-like) outer section. Thus, the elec- tron density seems to decrease from about $180 \mathrm{~cm}^{-3}$ at a distance of $1.5 \mathrm{kpc}$ from B1 to less than $10 \mathrm{~cm}^{-3}$ at about $4 \mathrm{kpc}$.

\section{THE ORIGIN OF THE LINER-LIKE IONIZATION}

Most regions of the Arp 299 system have been classified as LINER-like, although the mechanism producing this ionization needs to be determined. Some authors have found in ULIRGs a positive correlation between the $[\mathrm{O}$ I $] \lambda 6300 / \mathrm{H} \alpha$ ratio and the velocity dispersion (Monreal-Ibero et al. 2006), which has been considered as evidence supporting the shocks as the origin for the ionization (Armus et al. 1989; Dopita \& Sutherland 1995). The $\left[\mathrm{O}_{\mathrm{I}}\right] \lambda 6300 / \mathrm{H} \alpha$ versus the $\mathrm{H} \alpha$ velocity dispersion for all the spectra obtained with INTEGRAL has been represented (Fig. 13) in order to investigate the origin of the LINER-like ionization in Arp 299. The four main regions of the system observed with the INTEGRAL SB2 and SB3 bundles are analyzed separately: the inner and outer sections of the ionization cone, the interface region and finally the individual galaxies. The outer section of the ionization cone (LINER-like excitation, i.e., [O I] $\lambda 6300 / \mathrm{H} \alpha>$ -1.25 ; Fig. 13, stars in left panel) is independent of the velocity dispersion and presents a stable ionization level, while with a wider variety in the ionization level, the inner section of the cone (Seyfert-like; Fig. 13, triangles in left panel) does not follow the correlation founded in external regions of ULIRGs (MonrealIbero et al. 2006). The interface region (Fig. 13, crosses in left panel) presents a stable LINER-like excitation level, and is not dependent of the velocity dispersion.

With regard to the individual galaxies IC 694 and NGC 3690 as observed with the lower angular resolution (SB3) bundle (Fig. 13, filled circles in left panel), the ionization is mainly $\mathrm{H}$ IIlike, with no evidence of a kinematical correlation. For the higher angular resolution (SB2) data (Fig. 13, middle and right panels), the actual measurements of the individual galaxies do not follow the correlation, although they present a well-defined clustering. The galaxy IC 694 has a mean $\log \sigma \sim 1.8$, and presents about the same fraction of H II and LINER activity. In the case of NGC 3690 the velocity dispersion is clustered around $\log \sigma \sim 2$, and there is a large fraction of regions with $\mathrm{H}$ II-like ionization.

Clearly the present data show a behavior different than that found in ULIRGs (Monreal-Ibero et al. 2006), and this is probably due to the fact that on each case the measurements are done over regions located at different distances from the nucleus and with different characteristics. The original correlation was 
TABLE 5

Observed Magnitudes and Colors

\begin{tabular}{|c|c|c|c|c|c|}
\hline Region & $m_{\mathrm{F} 814 \mathrm{~W}}$ & $m_{\mathrm{F} 160 \mathrm{~W}}$ & $\mathrm{~F} 110 \mathrm{M}-\mathrm{F} 160 \mathrm{~W}$ & $\mathrm{~F} 160 \mathrm{~W}-\mathrm{F} 222 \mathrm{M}$ & F814W - F110M \\
\hline А & 16.85 & 13.72 & 1.33 & 1.36 & 1.80 \\
\hline 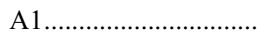 & 18.53 & 17.59 & 1.07 & 1.04 & -0.13 \\
\hline 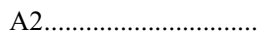 & 18.57 & 17.36 & 0.91 & 0.57 & 0.30 \\
\hline 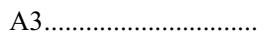 & 18.40 & 17.75 & 0.81 & 0.65 & -0.15 \\
\hline 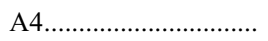 & 18.84 & 18.09 & 1.05 & 0.69 & -0.31 \\
\hline A5 & 18.19 & 17.61 & 0.72 & 0.63 & -0.14 \\
\hline 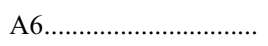 & 17.87 & 17.85 & 0.70 & 0.54 & -0.68 \\
\hline B1 & 15.67 & 13.93 & 1.23 & 2.06 & 0.51 \\
\hline 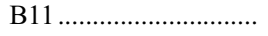 & 17.30 & 16.31 & 0.88 & 0.64 & 0.11 \\
\hline 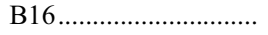 & 16.90 & 18.12 & 0.19 & $\ldots$ & -1.42 \\
\hline B2 & 14.09 & 13.19 & 0.70 & 0.69 & 0.20 \\
\hline B21 & 16.16 & 15.35 & 0.79 & 0.55 & 0.03 \\
\hline B22 & 17.78 & 16.49 & 0.93 & 0.75 & 0.36 \\
\hline B25 & 18.01 & 17.67 & 0.94 & 0.62 & -0.56 \\
\hline $\mathrm{C} 1$............................... & 17.04 & 14.68 & 1.71 & 2.67 & 0.66 \\
\hline $\mathrm{C} 2 \ldots \ldots \ldots \ldots \ldots \ldots \ldots$ & 16.63 & 16.57 & 0.67 & $\ldots$ & -0.61 \\
\hline 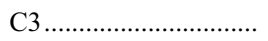 & 17.04 & 16.37 & 0.70 & 0.52 & -0.02 \\
\hline $\mathrm{C} 4 \ldots \ldots \ldots \ldots \ldots \ldots \ldots \ldots \ldots .$. & 17.24 & 16.71 & 0.82 & 0.44 & -0.29 \\
\hline D3 & 18.74 & 18.37 & 0.70 & 0.46 & -0.33 \\
\hline
\end{tabular}

NoтE.-Errors in the colors are \pm 0.10 and \pm 0.14 for the bright and faint sources, respectively.

derived for diffuse extranuclear regions in ULIRGs, which are located at distances of about $5-15 \mathrm{kpc}$ from the nucleus of the galaxy. In the present case the interface region and the ionization cone have regions at distances of up to $4 \mathrm{kpc}$ from the nucleus, and comparing with the original limits of the correlation (see Fig. 13, shaded area in left panel), the data of the interface and both regions of the ionization cone lie in general above the ratio, i.e., this regions show higher excitation for the same velocity dispersion. In the case of the individual galaxies observed with the actual higher resolution bundle (Fig. 13, middle and right panels), the present data are closer to the nucleus than in the case of ULIRGs (up to $\sim 2.5 \mathrm{kpc}$ ), and in most cases the regions involved are compact bright sources rather than diffuse. Comparing with the original limits of the relation (see Fig. 13, shaded areas in middle and right panels), the IC 694 data are clustered around, while the ones of NGC 3690 are below, the correlation. This means that in the present case for the individual galaxies the star formation influence is still present, and that the velocity dispersion close to the nucleus traces mass rather than flows/shocks.

Given an ionization mechanism, the ionization state of the surrounding gas can be changed as a result of the variations in the electron density, which affect the ionization parameter $(U \propto$ $N_{e}^{-1} r^{-2}$ ). This appears to be the case in the outer high-excitation cone associated with NGC 3690. As already mentioned, the electron density seems to have a radial variation within the cone, and by having the exact variation of $N_{e}$ with $r$, the radial dependence of parameter $U$ would be described more precisely. Unfortunately, in the present case the exact radial dependence of $N_{e}$ cannot be derived; in spite of that, the variation detected on the electron density, and therefore the LINER activity could be accounted for by the influence of an AGN (if $N_{e} \propto r^{-\alpha}$ ). The variation of $N_{e}$ implies a variation on the ionization parameter $\left(U \propto r^{-2+\alpha}\right)$, and thus the low excitation is valid if the source is not local.

In conclusion, for the Arp 299 system studied here there is no clear evidence for tidally induced shocks as found in ULIRGs. Therefore, the origin for the LINER ionization is unclear, and at least in some zones (i.e., outer parts of the cone) it could, perhaps, be due to a radiation field similar to that of a low-luminosity AGN.

\section{STAR FORMATION PROPERTIES}

It is well known that merging processes between gas rich galaxies can trigger star formation. As predicted by numerical simulations (Mihos \& Hernquist 1994), the process can vary depending on whether or not the parent disk galaxies have a bulge. The star-forming process at a low level (SFR $\sim 20 M_{\odot} \mathrm{yr}^{-1}$ ) consequence of the merging of two disk galaxies without a bulge is produced early on in the merging phase, and can be sustained for about 150 Myr. On the other hand, in the merger of galaxies with a bulge the starburst $\left(\mathrm{SFR} \sim 50-100 \mathrm{M}_{\odot} \mathrm{yr}^{-1}\right.$ ) is produced later in the merging phase, and lasts for about $50 \mathrm{Myr}$.

To investigate the properties of the young stellar populations of a galaxy assuming instantaneous and continuous bursts, synthetic models such as SB99 (Leitherer et al. 1999; Vazquez \& Leitherer 2005) can be used. In the following the stellar population synthesis model considers a Salpeter initial mass function (IMF) with lower and upper mass limits of 0.1 and $100 M_{\odot}$, the Geneva tracks with high mass loss, and the Calzetti extinction law (Calzetti et al. 2000). Instantaneous bursts are normalized to $10^{6} M_{\odot}$, while the continuous star formation is normalized to a star formation rate of $1 M_{\odot} \mathrm{yr}^{-1}$. Combining the information from the two-dimensional distribution of the $\mathrm{H} \alpha$ equivalent width and luminosity with the photometric results allows us to derive parameters such as the extinction to the gas, the age of the burst, and the stellar mass for each individual region. For the present analysis we use the INTEGRAL SB2 bundle with a resolution of 0 ." 9 (i.e., $0.18 \mathrm{kpc}$ ), and thus in the majority of cases the data include both the contribution from the stellar clusters and from a diffuse component.

\subsection{Photometry of the Regions of IC 694 and NGC 3690}

The aperture photometry as derived by AAH00, using the HST NIC1 F110M, NIC2 F160W, and NIC2 F222M images, is used in the following analysis. In addition, optical (WFPC2/ F814W) photometry over the same regions, using the same apertures and after performing the background subtraction and the aperture correction (for the latter see Holtzman et al. 1995), has been obtained. The apertures used were 0.9 diameter for the nuclei of IC 694 and 

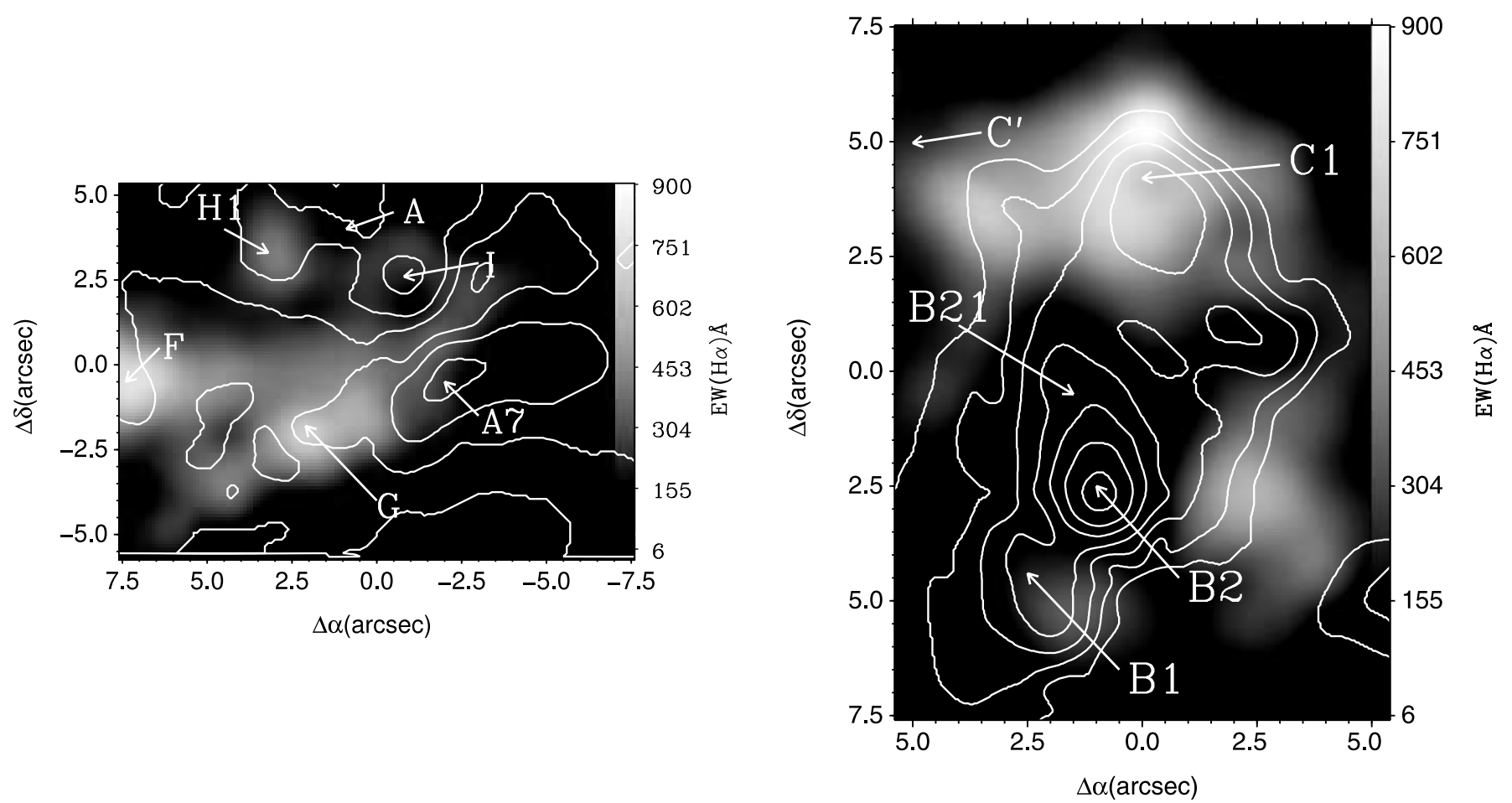

Fig. 14.- $\mathrm{H} \alpha$ equivalent width maps. North up, and east is to the left. Left: IC 694. Right: NGC 3690. Note that nuclei A and B1 have low equivalent widths. [See the electronic edition of the Journal for a color version of this figure.]

NGC $3690 \mathrm{~A}$ and B1, and for the star-forming region B2, and 0".5 diameter for the rest of the star-forming regions. The HST magnitudes and colors derived are shown in Table 5. In addition, the HST magnitudes and colors were modeled by convolving the simulated galaxy spectral energy distribution with the system (optics, filter, and detector) transmission function.

\subsection{Properties of the $\mathrm{H} \alpha$-emitting Regions}

In the following, instantaneous burst models characterized by the predicted $\mathrm{H} \alpha$ luminosity, predicted equivalent width, and near-IR colors are used to study the stellar populations of the $\mathrm{H} \alpha$ emitting regions under analysis. Comparing the measured values with the models, it is possible to derive the age, extinction, and mass of the individual regions under study. Regarding nucleus B1, and as previously indicated, it is classified as a Seyfert galaxy, and the fraction of the ionizing flux coming from the buried AGN in this region is not well determined. Therefore the conclusions regarding the star formation properties in $\mathrm{B} 1$ based on the $\mathrm{H} \alpha \mathrm{lu}-$ minosity have to be taken with caution, and represent an upper limit. Because of this fact, nucleus B1 is basically not included in the following discussion, although the properties derived are listed in the tables.

The $\mathrm{H} \alpha$ luminosity in specific regions with large $\mathrm{H} \alpha$ equivalent width (Fig. 14) traces preferentially the presence of young stellar populations $(<5-10 \mathrm{Myr})$. However, the presence of an old underlying population tends to decrease the EW and therefore leads to an underestimate of the age of the young stellar population. In the case of IC 694 and NGC 3690, the respective nuclei A and $\mathrm{B} 1$ present low $\mathrm{EW}(\mathrm{H} \alpha)$, as does the NGC 3690 brightest optical region $\mathrm{B} 2$. The ages derived from the $\mathrm{EW}(\mathrm{H} \alpha)$ for several individual regions are shown in Table 6 .

With an age for the nucleus (A) of $5.3 \times 10^{6} \mathrm{yr}$, IC 694 does not show a wide spread in age for the individual star-forming regions; almost all are around $5 \times 10^{6} \mathrm{yr}$, except for I1 with $5.9 \times$ $10^{6} \mathrm{yr}$ and the youngest regions, concentrated toward the east of the spiral-like arm ( $\mathrm{F}$ and $\mathrm{G}$, with 3.3 and $3.4 \times 10^{6} \mathrm{yr}$, respectively). The galaxy NGC 3690 shows an average age for the individual regions of $\sim 5.5 \times 10^{6} \mathrm{yr}$, with the oldest one $(7.2 \times$ $10^{6} \mathrm{yr}$ ) located toward the north of B2. The youngest sources are located in the northern part of the galaxy and correspond to complex C. Independent estimates (Satyapal et al. 1999), also based on instantaneous bursts and characterized by a Salpeter IMF, derived ages of about $6 \times 10^{6} \mathrm{yr}$ for the main components of the Arp 299 system, that are compatible with the results obtained here. Compared to AAH00, who used a different model with Gaussian bursts, the present age for B2 is younger, but applying their $\operatorname{EW}(\operatorname{Br} \gamma) \leq 5 \AA$ to our model, the age for B2 is $\geq 7 \times 10^{6} \mathrm{yr}$, compatible with the actual calculations.

Extinction and dust properties of the star-forming regions can be studied using the near-IR colors F110M - F160W (approximately $J-H$ ) and F160W - F222M (approximately $H-K$ ) together with the $\mathrm{EW}(\mathrm{H} \alpha)$. Under the assumption that the extinction to the stars is equivalent to the extinction toward the gas, the $\mathrm{EW}(\mathrm{H} \alpha)$ value is independent of $A_{V}$ (Fig. 15). We have unreddened both observed colors using a foreground screen model and the extinction derived from the $\mathrm{H} \alpha / \mathrm{H} \beta$ ratio, and compared with the models. Once corrected for extinction, and taking into account the error bars, in the majority of the regions the values are close to the predicted $A_{V}=2-3 \mathrm{mag}$ track. Thus in general the extinction derived from the $\mathrm{H} \alpha / \mathrm{H} \beta$ ratio presented in Tables 2 and 3 show an offset with respect to the ones predicted by the colors. There are two possible explanations for this discrepancy; on the one hand, the extinction derived from the $\mathrm{H} \alpha / \mathrm{H} \beta$ ratio could be underestimated (see $\S 5.4$ ); on the other hand the discrepancy may trace the contribution of hot dust emission. For the highly reddened regions (e.g., A, B1, ${ }^{10}$ and $\mathrm{C} 1$ ) the colors

\footnotetext{
${ }^{10}$ Nucleus B1 is included in this discussion related to the hot dust because even with high errors in the age derived from the $\operatorname{EW}(\mathrm{H} \alpha)$, the color excess exists and needs to be explained.
} 
TABLE 6

Properties of the Stellar Populations in Regions OF IC 694 AND NGC 3690

\begin{tabular}{|c|c|c|c|c|c|}
\hline Region & $\begin{array}{c}L(\mathrm{H} \alpha)^{\mathrm{a}} \\
\left(\times 10^{40} \mathrm{ergs} \mathrm{s}^{-1}\right)\end{array}$ & $\begin{array}{c}\mathrm{EW}(\mathrm{H} \alpha) \\
(\AA)\end{array}$ & $\begin{array}{l}\text { Age } \\
\left(\times 10^{6} \mathrm{yr}\right)\end{array}$ & $\begin{array}{r}\operatorname{Mass}\left(L_{\mathrm{H} \alpha}\right)^{\mathrm{b}} \\
\left(\times 10^{6} M_{\odot}\right)\end{array}$ & $\begin{array}{c}\operatorname{Mass}\left(M_{\mathrm{F} 160 \mathrm{~W}}\right)^{\mathrm{c}} \\
\quad\left(\times 10^{6} M_{\odot}\right)\end{array}$ \\
\hline A ................ & $9.2(150)$ & 201 & 5.3 & $40.0(655.0)$ & $200.0(460.0)$ \\
\hline A1 ........... & 4.8 & 217 & 5.2 & 17.6 & 6.0 \\
\hline A $2 \ldots \ldots \ldots . .$. & 4.6 & 292 & 5.0 & 13.0 & 6.4 \\
\hline A $3 \ldots \ldots \ldots . . . .$. & 3.2 & 297 & 5.0 & 9.0 & 4.8 \\
\hline $\mathrm{A} 4$.............. & 4.7 & 382 & 4.8 & 10.0 & 4.0 \\
\hline A $5 \ldots \ldots \ldots . . .$. & 7.3 & 307 & 5.0 & 21.0 & 5.3 \\
\hline A6........... & 6.1 & 197 & 5.3 & 26.8 & 5.0 \\
\hline A7 .............. & 11.3 & 277 & 5.1 & 36.5 & $\ldots$ \\
\hline$F \ldots \ldots \ldots . . .$. & 28.6 & 760 & 3.3 & 21.8 & $\ldots$ \\
\hline G................ & 7.9 & 654 & 3.4 & 6.2 & $\ldots$ \\
\hline $\mathrm{H} 1 \ldots \ldots \ldots . .$. & 18.6 & 416 & 4.7 & 34.0 & $\ldots$ \\
\hline $\mathrm{H} 2 \ldots \ldots \ldots$ & 22.5 & 314 & 4.9 & 57.0 & $\ldots$ \\
\hline $\mathrm{I} \ldots \ldots \ldots \ldots . . .$. & 5.8 & 220 & 5.2 & 21.5 & $\ldots$ \\
\hline I1 .............. & 1.6 & 86 & 5.9 & 12.9 & $\ldots$ \\
\hline B1 ........... & $62.3^{*}$ & $240^{*}$ & $5.2^{*}$ & $231.0^{*}$ & $200.0^{*}$ \\
\hline B2 ............. & 29.8 & 22 & 7.2 & 678.0 & 300.0 \\
\hline B11 ......... & 7.9 & 200 & 5.3 & 34.6 & 14.0 \\
\hline B16......... & 7.1 & 311 & 5.0 & 20.0 & 3.1 \\
\hline B21 ........ & 7.6 & 62 & 6.1 & 74.0 & 58.0 \\
\hline B22 ......... & 4.8 & 138 & 5.6 & 27.3 & 10.0 \\
\hline B25 ......... & 7.7 & 449 & 4.6 & 13.7 & 5.6 \\
\hline $\mathrm{C} 1 \ldots \ldots \ldots . . .$. & 93.8 & 680 & 3.4 & 74.0 & 81.0 \\
\hline $\mathrm{C} 2 \ldots \ldots \ldots$ & 53.4 & 645 & 3.5 & 43.8 & 9.8 \\
\hline $\mathrm{C} 3 \ldots \ldots \ldots$ & 9.7 & 440 & 4.7 & 17.7 & 9.8 \\
\hline $\mathrm{C} 4 \ldots \ldots \ldots . .$. & 2.1 & 156 & 5.4 & 10.3 & 8.4 \\
\hline C6........... & 6.8 & 609 & 3.6 & 5.7 & $\ldots$ \\
\hline $\mathrm{C}^{\prime} \ldots \ldots \ldots . .$. & $5.2(31.0)$ & 245 & 5.2 & $19.0(115.0)$ & $\ldots$ \\
\hline D3 ............. & 1.0 & 116 & 5.7 & 6.4 & 3.2 \\
\hline D4 ........... & 3.6 & 360 & 4.8 & 7.8 & $\ldots$ \\
\hline
\end{tabular}

${ }^{\mathrm{a}}$ Luminosity corrected for extinction using $\mathrm{H} \alpha / \mathrm{H} \beta$ ratio; the extinction using $\mathrm{Pa} \alpha / \mathrm{H} \alpha$ ratio is given for regions $\mathrm{A}$ and $\mathrm{C}^{\prime}$ in brackets.

${ }^{b}$ Mass derived from the $\mathrm{H} \alpha$ luminosity corrected by extinction using $\mathrm{H} \alpha / \mathrm{H} \beta$ ratio, and assuming the age listed in the fourth column. In brackets estimate using the $\mathrm{Pa} \alpha / \mathrm{H} \alpha$ extinction-corrected data.

c Mass derived from the absolute magnitude $M_{\mathrm{F} 160 \mathrm{~W}}$ corrected by extinction using $\mathrm{H} \alpha / \mathrm{H} \beta$ ratio, and assuming age listed in the fourth column. In brackets estimate using the $\mathrm{Pa} \alpha / \mathrm{H} \alpha$ extinction-corrected data. Astersisks indicated an AGN contribution.

measured (and corrected by extinction) are very red. As already mentioned (and discussed by AAH00), one of the most plausible explanations for this color excess is the presence of hot dust $(T>600 \mathrm{~K})$, which would mainly affect the F160W - F222M color. In an attempt to take into account the contribution of the hot dust emission, the $\lambda^{-2} B_{\lambda}$ wavelength modified blackbody thermal emission (Hildebrand 1983) has been used. The results demonstrate that depending on the temperature (which need not necessarily be homogeneous over the line of sight) and the amount of dust, the contribution of hot dust $(\sim 800 \mathrm{~K})$ emission could represent, on average, about $80 \%$ of the $K$-band emission (Fig. 15 , right panel) for the highly extincted regions (A, B1, and $\mathrm{C} 1$ ), and about $30 \%$ for the rest of the regions. This result supports our previous conclusion that the dust is more concentrated toward the nuclei and some particular regions than in the extranuclear starforming regions.

An alternative to the presence of a hot dust emission is that the structure of the dust-gas distribution does not follow the simple screen geometry, but it is closer to a clumpy foreground or a mixed dust-star distribution. Assuming a model where the gas is homogeneously mixed with the stars, the effect of an $A_{V}=6$ mag over the F160W - F222M and F110M - F160W measured colors will produce bluer colors, about 0.3 and $0.4 \mathrm{mag}$, respectively (Scoville et al. 2000; see Fig. 15). For the extreme case $A_{V}=30$, the corresponding shifts will be 0.6 and $0.8 \mathrm{mag}$; i.e., in region $\mathrm{C} 1$ the F160W - F222M color will move from the observed 2.67 to 2.07 . Thus the colors modeled with this alternative dust geometry would imply that a hot dust contribution is not mandatory in the external, less extincted regions, but is still required to explain the color excess in the highly extincted regions $(\mathrm{A}, \mathrm{B} 1$, and $\mathrm{C} 1)$.

The stellar mass of the young population has been measured using two different indicators, the $\mathrm{H} \alpha$ luminosity and the $\mathrm{M}_{160 \mathrm{w}}$ absolute magnitude, and comparing with the predicted values for the ages derived using the $\mathrm{EW}(\mathrm{H} \alpha)$. In both cases the $\mathrm{H} \alpha / \mathrm{H} \beta$ ratio has been used to correct for extinction (for $\mathrm{A}$ and $\mathrm{C}^{\prime}$ the $\mathrm{Pa} \alpha /$ $\mathrm{H} \alpha$ ratio was used too).

The results obtained using $M_{160 \mathrm{~W}}$ (see Fig. 16 and Table 6) show that the regions with the most young stellar mass content are nucleus A and regions B2, B21, and $\mathrm{C} 1(460,300,58$, and $81 \times 10^{6} M_{\odot}$, respectively). The stellar mass content for the rest of the regions, including the low surface brightness knots, ranges between 3.1 and $14.0 \times 10^{6} M_{\odot}$ (details in Table 6). Previous derivations of the stellar mass of the clusters used absolute $H$-band magnitudes versus $H-K$ (not corrected for extinction) with a different stellar population synthesis model range, from about $1.5 \times 10^{5}$ to $5.0 \times 10^{6} M_{\odot}(\mathrm{AAH} 00)$.

Using the $\mathrm{H} \alpha$ luminosity, the young stellar mass content is again concentrated in nucleus A and regions B2, B21, and C1 $\left(655,679,74\right.$, and $74 \times 10^{6} M_{\odot}$, respectively). For region $\mathrm{C}^{\prime}$, for which we do not have near-IR photometry, the mass derived using the $\mathrm{H} \alpha$ luminosity is $115 \times 10^{6} M_{\odot}$. The rest of the derived masses range from 5.7 to $57 \times 10^{6} M_{\odot}$. Comparing the mass in newly formed stars with the derivations of AAH00, there are no significant differences except for B2, where we derive a value 2 times higher. The masses of the young $\mathrm{H} \alpha$-emitting regions identified in Arp 299 are within the range of masses founded in LIRGs $\left(3 \times 10^{6}-10^{8} M_{\odot}\right.$; Scoville et al. 2000; Alonso-Herrero et al. 2002) and are on average about 100 times more massive than the galactic globular clusters $\left(10^{5} M_{\odot}\right.$; van den Bergh 1995), probably due to the fact that the present regions have conglomerates of less massive clusters.

When comparing the stellar masses derived with the $\mathrm{H} \alpha$ luminosity with the mass obtained from the absolute F160W magnitude, there is a good agreement in nucleus A, but for the rest of the regions there are differences from a factor $\sim 1$ to 5 . One of the main reasons for this difference is that in the majority of the cases (except A and B2) an aperture of 0."5 has been used for the $H S T$ cluster photometry, instead the 0.9 or 1 ." 0 used for the INTEGRAL data analysis [for the $\mathrm{EW}(\mathrm{H} \alpha)$ and $\mathrm{L}(\mathrm{H} \alpha)$ estimates]; this difference can lead to offsets of a factor of 3 in the mass estimates. Factoring in the aperture effects, the two methods give the same mean value within a factor of 2 .

\subsection{Contribution to the Bolometric Luminosity}

The derived ages of the young $\mathrm{H} \alpha$-emitting star-forming regions cover a range from $3 \times 10^{6}$ to at least $(7-8) \times 10^{6}$ yr old. This is consistent with the methodology used here: since the $\mathrm{H} \alpha$ traces preferentially the massive young ionizing stellar clusters, populations older than $10 \mathrm{Myr}$ become more difficult to detect as $\mathrm{H} \alpha$ decreases. In any case, there is further evidence that the star formation in Arp 299 has persisted for at least the last (10-15) $\times$ $10^{6} \mathrm{yr}$ (AAH00), with high activity in the star-forming regions. On the basis of this dispersion, we conclude that the overall star formation of Arp 299 can be modeled by a continuous star formation. Under this premise, the star formation rate (SFR) and the 

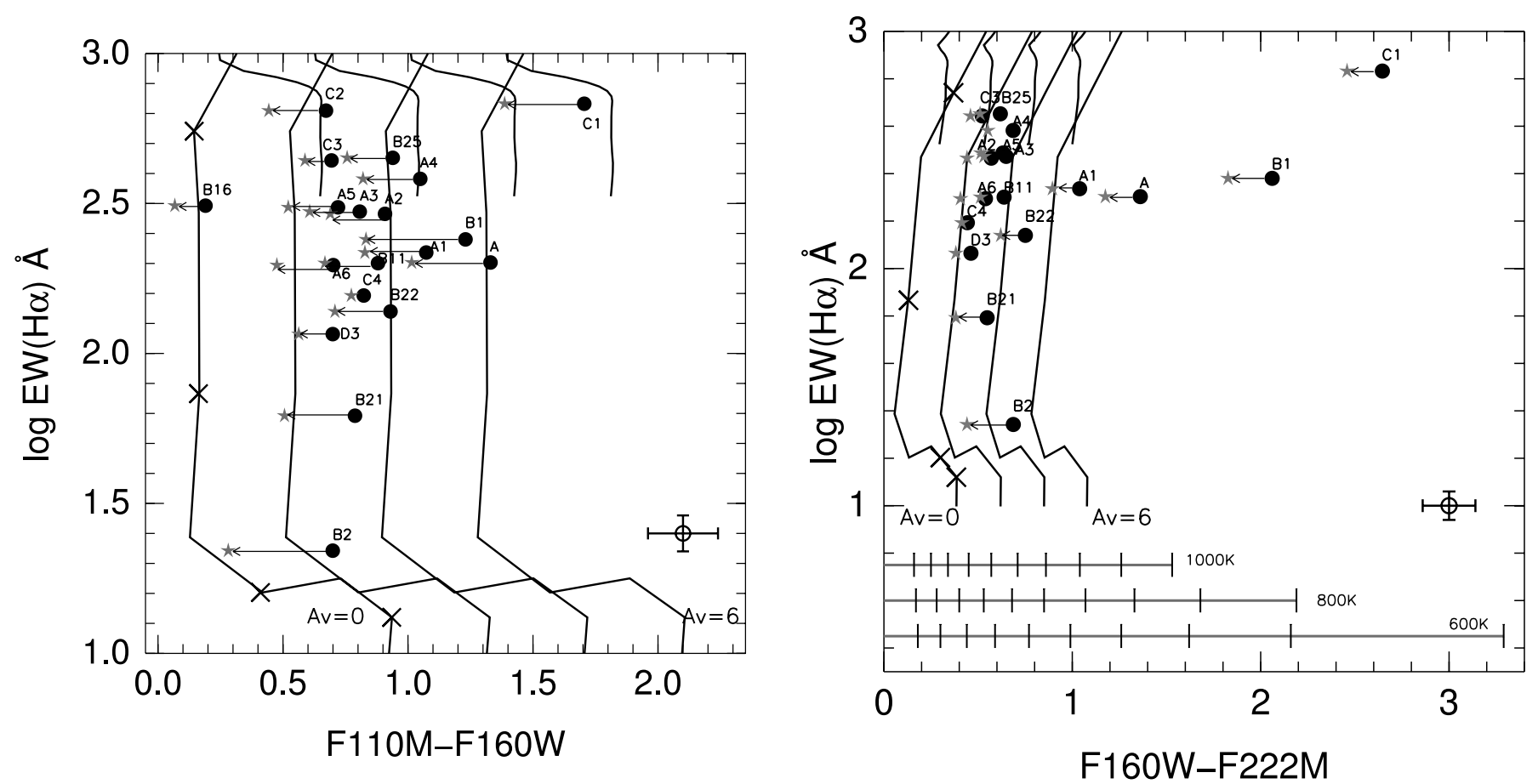

FIG. 15.-Plots representing the results of the stellar evolutionary models (SB99, Geneva high isochrones, Salpeter IMF, using upper and lower mass boundaries of 0.1 and $100 M_{\odot}$ ) compared with the measured values. The long tracks represent the evolution of instantaneous bursts as a function of age (from top to bottom, black crosses mark 4, 6 , 8 , and $10 \mathrm{Myr}$ ). Short tracks correspond to continuous bursts using a SFR of $1 M_{\odot} \mathrm{yr}^{-1}$. In both cases from left to right the extinction ranges from $A_{V}=0$ to $A_{V}=6$, in steps of 2 mag. The filled circles show the measured values, and the stars represent the extinction-corrected values from the $\mathrm{H} \alpha / \mathrm{H} \beta$ ratio. Left: F110M $-\mathrm{F} 160 \mathrm{~W}$ color vs. H $\alpha$ equivalent width. Right: F160W - F222M color vs. H $\alpha$ equivalent width. The long horizontal lines represent the colors expected from a extinction-free stellar population of $6 \times 10^{6} \mathrm{yr}$ with an increasing $K$-band flux contribution of hot dust at different temperatures (from $0 \%$ to $100 \%$, increasing $10 \%$ on each step). In both cases nucleus B1 is included for completeness. [See the electronic edition of the Journal for a color version of this figure.]

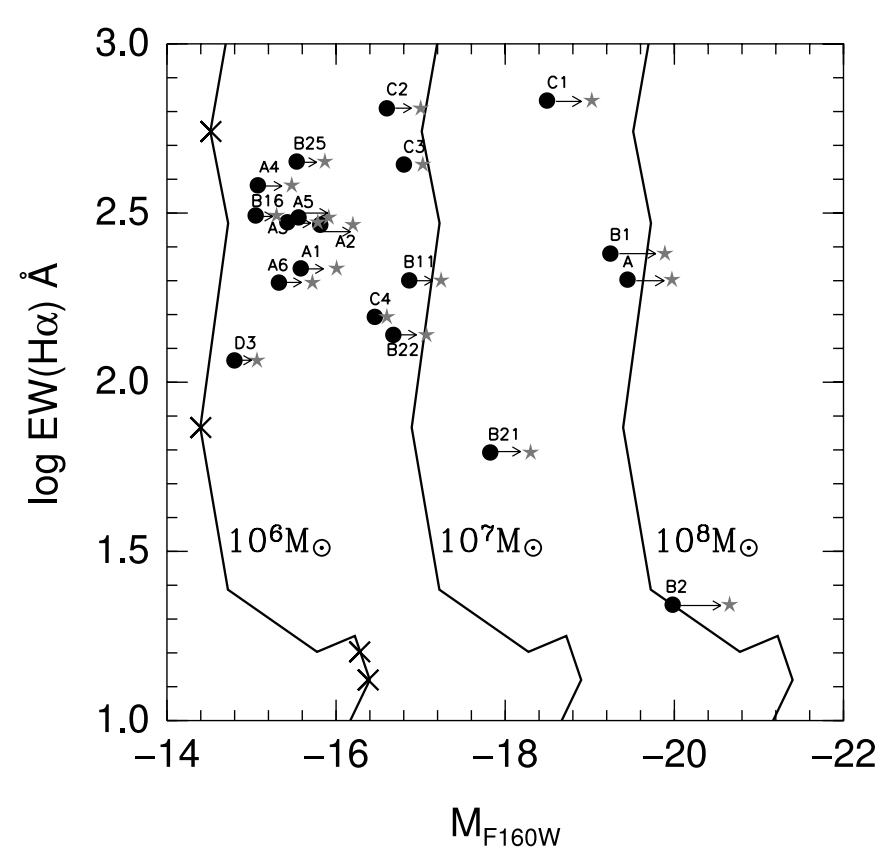

Fig. 16.-Results of the stellar evolutionary models (SB99, Geneva high isochrones, Salpeter IMF, using upper and lower mass boundaries of 0.1 and $100 M_{\odot}$ ) compared with measured values. Long tracks are the output for instantaneous bursts as a function of age (from top to bottom, black crosses mark 4, 6, 8, and $10 \mathrm{Myr}$ ), and different cluster mass. The filled circles represent measured absolute F160W magnitude, and the stars represent the extinction-corrected values from the $\mathrm{H} \alpha / \mathrm{H} \beta$ ratio. [See the electronic edition of the Journal for a color version of this figure.]
IR luminosity contribution can be derived from the extinctioncorrected H $\alpha$ luminosity, using Kennicutt's (1998) expressions, which are valid for a Salpeter IMF with mass cutoffs of $0.1-100 M_{\odot}$ :

$$
\begin{gathered}
\operatorname{SFR}\left(M_{\odot} \mathrm{yr}^{-1}\right)=7.9 \times 10^{-42} L(\mathrm{H} \alpha)\left(\mathrm{erg} \mathrm{s}^{-1}\right), \\
L_{\mathrm{IR}}\left(\mathrm{ergs} \mathrm{s}^{-1}\right)=175.5 \times L(\mathrm{H} \alpha)\left(\mathrm{ergs} \mathrm{s}^{-1}\right),
\end{gathered}
$$

which strictly speaking are only valid for starbursts with ages less than $10^{8} \mathrm{yr}$.

Without taking into account nucleus B1 (an AGN candidate), the SFR of the entire system is about $43 M_{\odot} \mathrm{yr}^{-1}$, and the $L_{\mathrm{IR}}$ is $9.5 \times 10^{44} \mathrm{ergs} \mathrm{s}^{-1}$, whereas the luminosity derived from the IRAS fluxes is $2.2 \times 10^{45} \mathrm{ergs} \mathrm{s}^{-1}$, and thus the contribution of the studied regions to the IR luminosity is about $45 \%$. There is a gap of about $55 \%$ in luminosity, which can be accounted for by the AGN located in B1. Previous estimates based on the mid- and far-IR spectra of Arp 299 assign a $20 \%$ of the $L_{\mathrm{IR}}$ coming from B1 and B2 (Charmandaris et al. 2002), while at $11.7 \mu \mathrm{m}$ the percentage of flux coming from B1 and B2 is about 30\% (Keto et al. 1997). So even considering that the majority of this contribution comes from $\mathrm{B} 1$, there is still a gap of about $25 \%-35 \%$. This inconsistency is probably due to the fact that the present result is limited by the derived extinction and/or the models.

\section{TWO-DIMENSIONAL GAS KINEMATICS AND DYNAMICAL MASS DERIVATIONS}

Previous studies of Arp 299 (Gehrz et al. 1983; Telesco et al. 1985) concluded that this system consists of two spiral galaxies disturbed by the merging process, which are rotating in opposite sense (Augarde \& Lequeux 1985). More recently, Hibbard \& 
TABLE 7

Kinematic of the Interface Region

\begin{tabular}{|c|c|c|c|}
\hline Region & $\begin{array}{c}V(\mathrm{H} \alpha)^{\mathrm{a}} \\
\left(\mathrm{km} \mathrm{s}^{-1}\right)\end{array}$ & $\begin{array}{l}V(\mathrm{Na} \mathrm{I})^{\mathrm{b}} \\
\left(\mathrm{km} \mathrm{s}^{-1}\right)\end{array}$ & $\begin{array}{l}\sigma(\mathrm{H} \alpha)^{\mathrm{c}} \\
\left(\mathrm{km} \mathrm{s}^{-1}\right)\end{array}$ \\
\hline $\mathrm{K} 1 \ldots \ldots \ldots . .$. & -56 & $-29:$ & 61 \\
\hline $\mathrm{K} 2 \ldots \ldots \ldots$ & -32 & $\ldots$ & 58 \\
\hline $\mathrm{K} 3 \ldots \ldots \ldots$ & -65 & 30 & 80 \\
\hline $\mathrm{K} 4 \ldots \ldots \ldots \ldots$ & 13 & 31 & 68 \\
\hline$\ldots \ldots \ldots \ldots \ldots \ldots$ & 54 & 101 & 35 \\
\hline 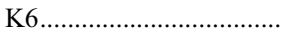 & 54 & 115 & 20 \\
\hline 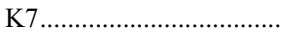 & 36 & 78 & 30 \\
\hline 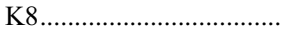 & 84 & 42 & 70 \\
\hline 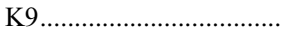 & 20 & $-6:$ & 65 \\
\hline 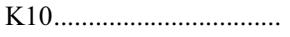 & 54 & 86: & 100 \\
\hline 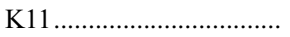 & -2 & 26 & 19 \\
\hline 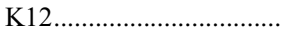 & -78 & $\ldots$ & 36 \\
\hline
\end{tabular}

Notes. - The mean errors for the velocity measurements in $\mathrm{H} \alpha$ and $\mathrm{Na}$ I for this bundle are $27 \mathrm{~km} \mathrm{~s}^{-1}$ and $60 \mathrm{~km} \mathrm{~s}^{-1}$, respectively. a colon means large uncertainties. Velocities referred to nucleus (region A) of galaxy IC 694.

${ }^{\mathrm{a}} V_{A}(\mathrm{H} \alpha)=3121 \pm 27 \mathrm{~km} \mathrm{~s}^{-1}$.

b $V_{A}(\mathrm{Na} \mathrm{I})=3057 \pm 34 \mathrm{~km} \mathrm{~s}^{-1}$.

c Relative error in $\sigma$ is $\simeq 13 \%$.

Yun (1999) have described Arp 299 as a structure that formed after a prograde-retrograde encounter between IC $694(\mathrm{Sab}-\mathrm{Sb})$ and NGC 3690 (Sbc-Sc) that took place some $750 \mathrm{Myr}$ ago and has a long tidal tail (180 kpc in length) detected in H I. Although there are still two clearly distinguishable structures with the two nuclei separated by about $4.6 \mathrm{kpc}$, the warm gas component has lost its kinematic identity and the velocity field presents a very irregular pattern (Rampazzo et al. 2005).

\subsection{Gas Kinematics}

The kinematics of the ionized and the neutral gas is traced by the $\mathrm{H} \alpha$ emission line and the $\mathrm{Na}$ I $\lambda 5890$ absorption line, respectively (Sparks et al. 1997). The ionized gas mean velocities and velocity dispersions were obtained by fitting the $\mathrm{H} \alpha+[\mathrm{N} \mathrm{II}]$ $\lambda \lambda 6548,6584$ complex with a single Gaussian velocity component per line; the same method was applied to the He I $25876+$ $\mathrm{Na}$ I $\lambda \lambda 5890,5896$ complex. The values derived for the indi-

TABLE 8

Kinematic of IC 694 Regions

\begin{tabular}{|c|c|c|c|}
\hline Region & $\begin{array}{l}V(\mathrm{H} \alpha)^{\mathrm{a}} \\
\left(\mathrm{km} \mathrm{s}^{-1}\right)\end{array}$ & $\begin{array}{l}V(\mathrm{Na} \mathrm{I})^{\mathrm{b}} \\
\left(\mathrm{km} \mathrm{s}^{-1}\right)\end{array}$ & $\begin{array}{l}\sigma(\mathrm{H} \alpha)^{\mathrm{c}} \\
\left(\mathrm{km} \mathrm{s}^{-1}\right)\end{array}$ \\
\hline А & 0 & 0 & 108 \\
\hline A1 & -86 & -47 & 73 \\
\hline A2 & -7 & 22 & 64 \\
\hline A3 & -134 & -86 & 36 \\
\hline A4 & -151 & -96 & 50 \\
\hline 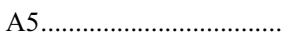 & -67 & -25 & 45 \\
\hline 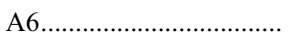 & -44 & -37 & 40 \\
\hline 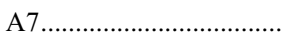 & -57 & -26 & 41 \\
\hline F & -163 & $\ldots$ & 72 \\
\hline 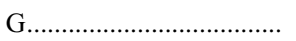 & -121 & -77 & 43 \\
\hline 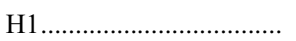 & -130 & -89 & 61 \\
\hline $\mathrm{H} 2$ & -117 & -93 & 109 \\
\hline I & 60 & 98 & 61 \\
\hline 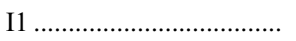 & 37 & $\ldots$ & 79 \\
\hline
\end{tabular}

Note.-Velocities referred to nucleus (region A).

${ }^{\mathrm{a}} V_{A}(\mathrm{H} \alpha)=3121 \pm 27 \mathrm{~km} \mathrm{~s}^{-1}$

b $V_{A}(\mathrm{Na} \mathrm{I})=3057 \pm 34 \mathrm{~km} \mathrm{~s}^{-1}$.

${ }^{c}$ Relative error in $\sigma$ is $\simeq 10 \%$.
TABLE 9

Kinematic of NGC 3690 Regions

\begin{tabular}{|c|c|c|c|}
\hline Region & $\begin{array}{c}V(\mathrm{H} \alpha)^{\mathrm{a}} \\
\left(\mathrm{km} \mathrm{s}^{-1}\right)\end{array}$ & $\begin{array}{l}V(\mathrm{Na} \mathrm{I})^{\mathrm{b}} \\
\left(\mathrm{km} \mathrm{s}^{-1}\right)\end{array}$ & $\begin{array}{c}\sigma(\mathrm{H} \alpha)^{\mathrm{c}} \\
\left(\mathrm{km} \mathrm{s}^{-1}\right)\end{array}$ \\
\hline B1 & 0 & 0 & 172 \\
\hline 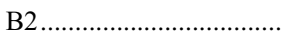 & -116 & -55 & 110 \\
\hline 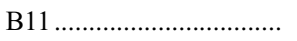 & 38 & 50 & 143 \\
\hline 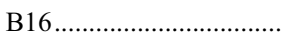 & -46 & -15 & 117 \\
\hline 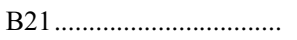 & -18 & 9 & 128 \\
\hline 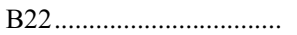 & -121 & -48 & 78 \\
\hline В25 & -112 & -64 & 54 \\
\hline $\mathrm{C} 1$ & 32 & 65 & 93 \\
\hline $\mathrm{C} 2$ & 16 & 51 & 99 \\
\hline $\mathrm{C} 3$ & 13 & 36 & 90 \\
\hline $\mathrm{C} 4$ & -24 & 8 & 94 \\
\hline С6 & 115 & $\ldots$ & 82 \\
\hline $\mathrm{C}^{\prime}$ & 101 & $\ldots$ & 69 \\
\hline D3 & -101 & -40 & 67 \\
\hline D4 & -112 & -52 & 54 \\
\hline
\end{tabular}

Note.-Velocities referred to nucleus (regions B1).

${ }^{\mathrm{a}} V_{B 1}(\mathrm{H} \alpha)=3040 \pm 27 \mathrm{~km} \mathrm{~s}^{-1}$.

b $V_{B 1}(\mathrm{Na} \mathrm{I})=2976 \pm 32 \mathrm{~km} \mathrm{~s}^{-1}$.

${ }^{\mathrm{c}}$ Relative error in $\sigma$ is $\simeq 10 \%$.

vidual regions are presented in Tables 7, 8, and 9. For the velocity measurements, the errors have been calculated taking into account both the systematic uncertainties of the wavelength calibration (the same for all lines) in the velocity measurement and the uncertainties in the measurement of the centroid of the line (which depends on the signal-to-noise ratio of the studied line). One of the most relevant results obtained is that the twodimensional structure of the ionized and neutral gas velocity fields show similar features in both the individual galaxies and the interface region, indicating that the ionized and neutral gas kinematics are coupled.

The structure of the $\mathrm{H} \alpha$ velocity dispersion shows that there is a variation on the average velocity dispersion values: the interface region has the lowest $\left(45 \mathrm{~km} \mathrm{~s}^{-1}\right)$, IC 694 the intermediate $\left(65 \mathrm{~km} \mathrm{~s}^{-1}\right)$, and NGC $3690\left(120 \mathrm{~km} \mathrm{~s}^{-1}\right)$ the maximum values of $\sigma$.

\subsubsection{Interface Region}

The overall velocity field of the ionized gas as observed with the SB3 bundle has a complex and irregular pattern that does not indicate the presence of virialized simple (e.g., rotating) motions, on scales of $0.6 \mathrm{kpc}$ or larger (Fig. 17, left). The structure of this map agrees well with that obtained by Rampazzo et al. (2005) covering a larger area (FOV of 5.'9) on the sky. The velocities in the northern part are receding, and those in the southern part are approaching the systemic value, defined as the velocity in nucleus A (the dust-enshrouded nucleus of IC 694) with the higher spatial resolution bundle SB2, $V_{A}=3121 \pm 27 \mathrm{~km} \mathrm{~s}^{-1}(\operatorname{see} \S 10.1 .2)$. The corresponding lower spatial resolution SB3 velocity is $V_{A}=3190 \pm 27 \mathrm{~km} \mathrm{~s}^{-1}$.

The velocity field of the neutral gas obtained from the Na D absorption lines, despite of being noisier (the intensity of the $\mathrm{NaD}$ can be typically $2 \%$ of that of $\mathrm{H} \alpha$ ), has structural characteristics similar to those of the ionized gas velocity field (Fig. 17, center panel), and presents a systemic velocity of $3057 \pm 34 \mathrm{~km} \mathrm{~s}^{-1}$.

The ionized gas velocity field presents a peak-to-peak velocity of about $230 \mathrm{~km} \mathrm{~s}^{-1}$, while for the neutral gas the variation is smaller, about $170 \mathrm{~km} \mathrm{~s}^{-1}$. In both cases the area with the largest approaching velocities is close to $\mathrm{B} 2$. The ionization cone does 

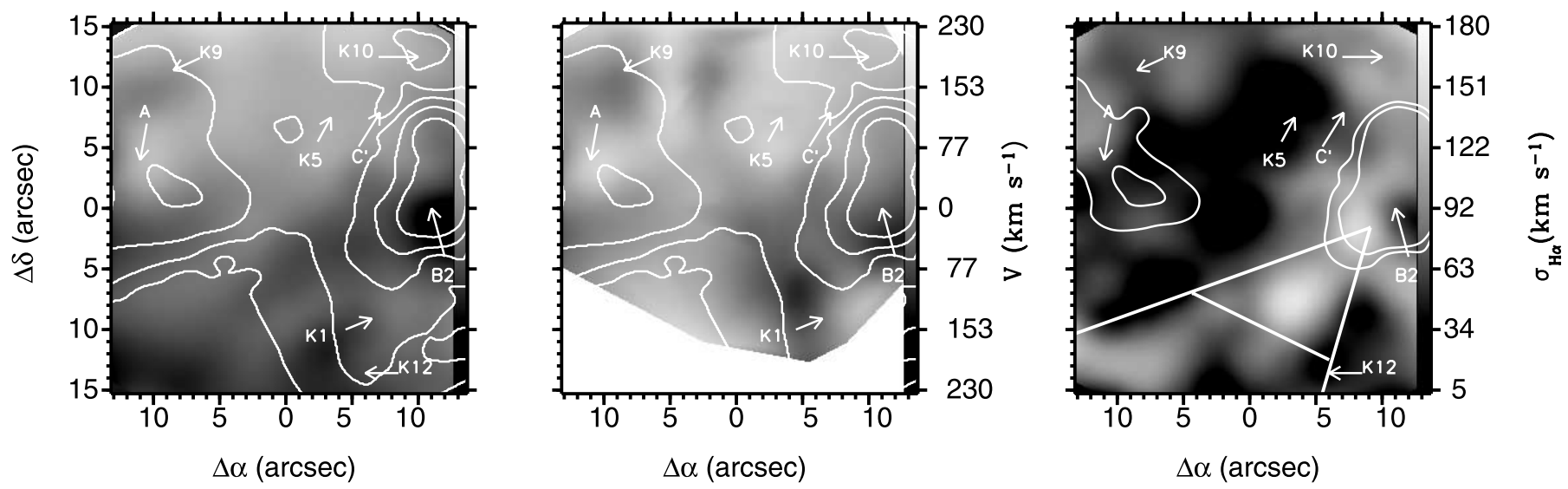

FIG. 17.-Arp 299 interface region kinematics. Velocity field of the ionized gas as traced by the H $\alpha$ line (left), neutral gas as traced by the Na I line (middle), and velocity

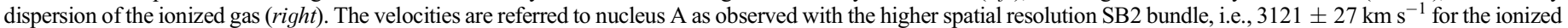
and $3057 \pm 34 \mathrm{~km} \mathrm{~s}^{-1}$ for the neutral gas. Lines depicted the ionization cone as in Fig 10. [See the electronic edition of the Journal for a color version of this figure.]

not show relevant features in the ionized gas velocity field, nor in the neutral gas velocity field. With the exception of the nuclear regions, the ionized gas in the galaxies does not have a distinct kinematics with respect to the interface region (i.e., the structure of the velocity field does not correlate with the distribution of mass as traced by the continuum images).

The velocity gradient of the ionized gas through the interface is about $40 \mathrm{~km} \mathrm{~s}^{-1} \mathrm{kpc}^{-1}$, compatible with the $\mathrm{H} \alpha$ velocity gradients previously measured across ULIRGs observed with a slit (up to $47 \mathrm{~km} \mathrm{~s}^{-1} \mathrm{kpc}^{-1}$ for galaxies with double nuclei, Martin 2005).

The velocity dispersion shows distinct features over the entire area; there are large differences in $\sigma$, that allow us to discriminate spatially between quiet and turbulent gas with macroscopic motions (Fig. 17, right panel). The so-called interface has an average $\sigma$ value of $45 \mathrm{~km} \mathrm{~s}^{-1}$ that traces no turbulent gas. The low velocity amplitude and velocity dispersion of the interface indicates that the ionized gas could be in a close to quiescent phase or slowly rotating. Larger $\sigma$ values are measured for the previously defined ionization cone, with the maximum $\left(160 \pm 21 \mathrm{~km} \mathrm{~s}^{-1}\right)$ located on the peak of Seyfert-like ionization. This spatial coincidence could indicate that in that area the two contributions of the biconic surface of the cone are added, and thus the line measured is wider (Heckman et al. 1990). On the other hand, the maximum velocity dispersion would imply that the largest macroscopic motions, with approaching velocities, are taking place in that highly ionized area.

\subsubsection{IC 694}

The velocity field of both the ionized and neutral gas presents a similar structure (Fig. 18, left and center panels) when observed with the SB2 bundle. It has an irregular structure, with the largest receding velocities associated with region I (3181 \pm $27 \mathrm{~km} \mathrm{~s}^{-1}$ for the ionized gas and $3155 \pm 34 \mathrm{~km} \mathrm{~s}^{-1}$ for the neutral), located $0.42 \mathrm{kpc}$ southwest of nucleus A. These maps show a general trend with velocities changing from northwest (receding) to southeast (approaching), in agreement with the pattern found with the SB3 data in this region. The systemic velocity for the ionized gas is defined as the velocity associated with the nucleus (region A), and it has a value of $3121 \pm 27 \mathrm{~km} \mathrm{~s}^{-1}$, consistent with Zhao et al. (1997) estimate based on the H92 $\alpha$ radio recombination line $\left(3120 \pm 50 \mathrm{~km} \mathrm{~s}^{-1}\right)$. The ionized gas velocity field has a peak-to-peak variation of $290 \mathrm{~km} \mathrm{~s}^{-1}$. The neutral gas systemic velocity is $3057 \pm 34 \mathrm{~km} \mathrm{~s}^{-1}$, and the peakto-peak velocity is about $216 \mathrm{~km} \mathrm{~s}^{-1}$. Although evident structural similarities are found between the ionized and neutral gas (i.e., same kinematics), in general the velocity of the neutral gas presents a blueshift over the entire area with respect to the ionized gas (about $-30 \pm 12 \mathrm{~km} \mathrm{~s}^{-1}$ over the studied regions). This systematic blueshift appears to be real, taking into account the uncertainties. In that case, this is a marginal effect, with a lower value than the ones measured in ULIRGs using a slit (Martin 2005 ; from about -60 to $-300 \mathrm{~km} \mathrm{~s}^{-1}$ ). In the present analysis the value obtained is derived from IFS data, i.e., has the
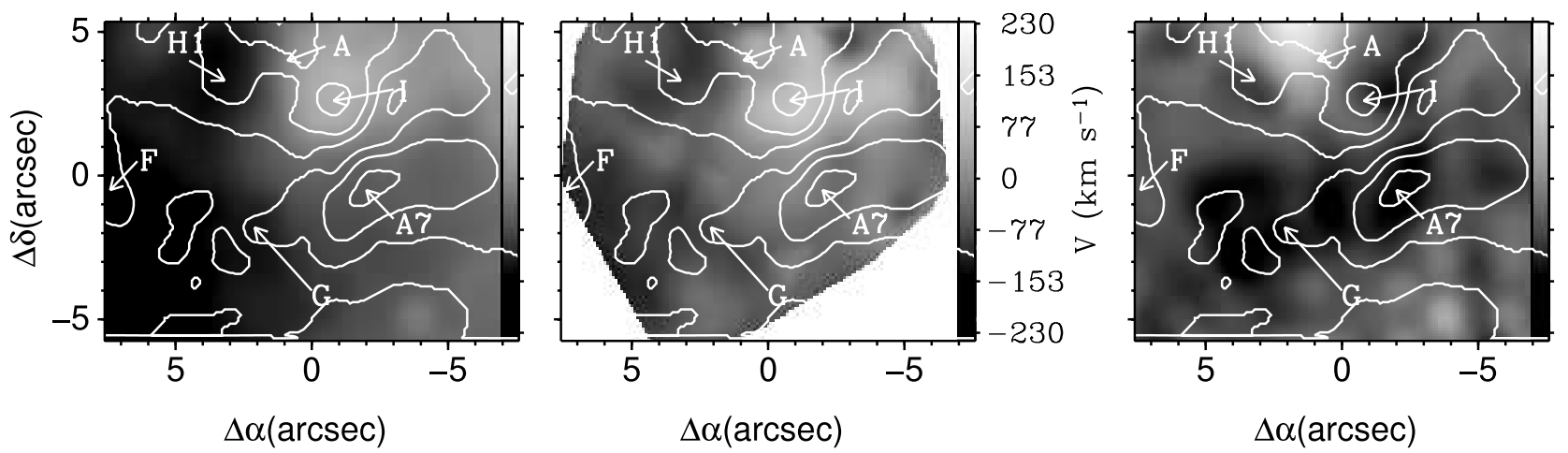

180

151 122 is

92

63

돌

34

5

FIG. 18.- IC 694 kinematics. Velocity field of the ionized gas as traced by the H $\alpha$ line (left), neutral gas as traced by the Na I line ( $m i d d l e$ ), and velocity dispersion of

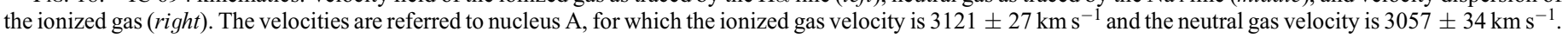
[See the electronic edition of the Journal for a color version of this figure.] 

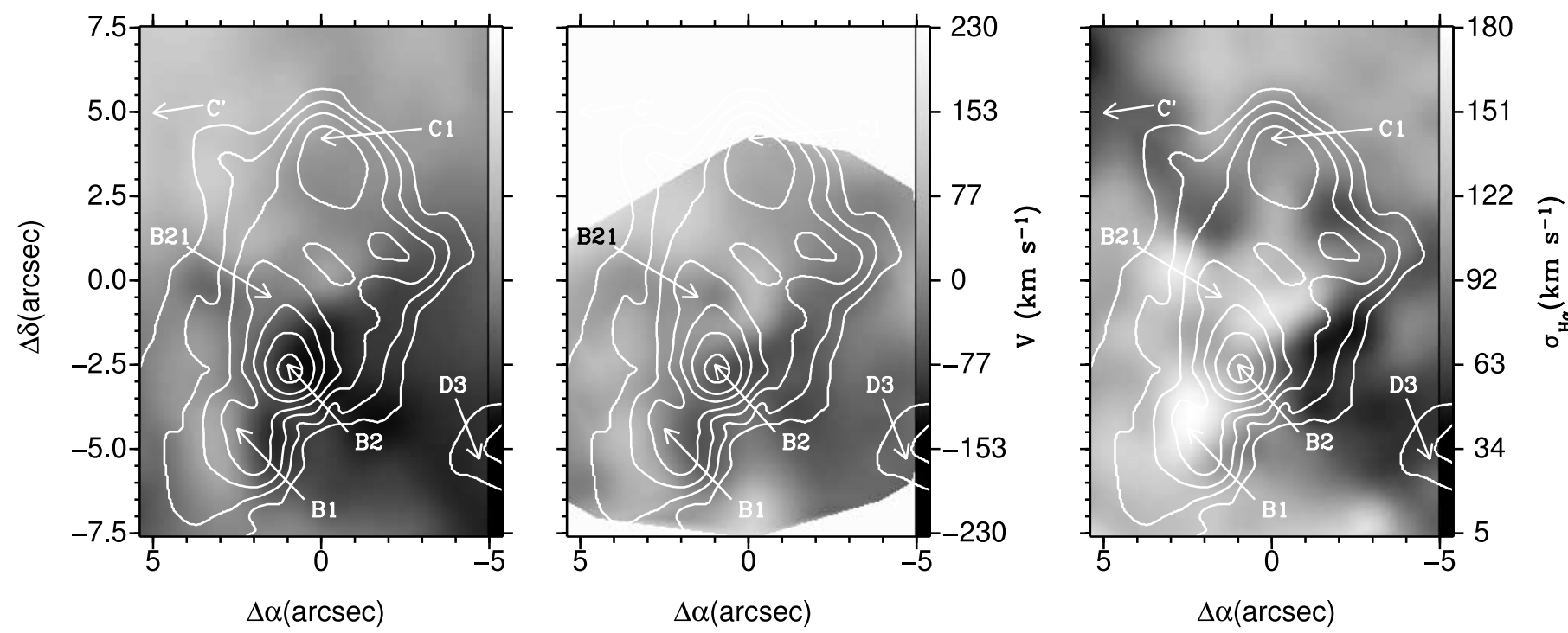

FIG. 19. - NGC 3690 kinematics. The velocity field of the ionized gas as traced by the $\mathrm{H} \alpha$ line (left), neutral gas as traced by the Na I line (middle), and velocity dispersion of the ionized gas (right). The velocities are referred to nucleus B1, for which the ionized gas velocity is $3040 \pm 27 \mathrm{~km} \mathrm{~s}^{-1}$ and the neutral gas velocity is $2976 \pm 32 \mathrm{~km} \mathrm{~s}^{-1}$. Note that nucleus B1 presents the highest $\sigma$ value. [See the electronic edition of the Journal for a color version of this figure.]

advantage of using the two-dimensional distribution rather than the slit, where the measurements are limited to a restricted area, probably centered on the nuclear regions.

Across the galaxy there are gradients of about $110 \mathrm{~km} \mathrm{~s}^{-1}$ $\mathrm{kpc}^{-1}$ in the ionized gas velocity (between regions located $\sim 2.3 \mathrm{kpc}$ apart i.e., $\sim 11^{\prime \prime}$ ), higher than the typical values founded in ULIRGs (up to $60 \mathrm{~km} \mathrm{~s}^{-1} \mathrm{kpc}^{-1}$ for an individual galaxy; Martin 2005 ) when using a slit of $20^{\prime \prime}$ long. In closer regions with large velocity variations the gradients can be $150 \mathrm{~km} \mathrm{~s}^{-1} \mathrm{kpc}^{-1}$ (between regions I and northern of $\mathrm{G}$, located about $1.4 \mathrm{kpc}$ apart). In spite of these gradients, and as discussed below, there is no clear evidence for virialized simple rotating motions.

The two-dimensional velocity dispersion map shows a different structure than the velocity field (Fig. 18, right panel). For nucleus A, the $\sigma$ value is $108 \pm 11 \mathrm{~km} \mathrm{~s}^{-1}$, in good agreement with the derivation of Shier et al. (1996) based on the CO $(2,0)$ nearinfrared band at $2.3 \mu \mathrm{m}\left(135 \pm 21 \mathrm{~km} \mathrm{~s}^{-1}\right)$. The maximum value for $\sigma\left(170 \pm 20 \mathrm{~km} \mathrm{~s}^{-1}\right)$ is located in a region $0.28 \mathrm{kpc}$ toward the northeast of $\mathrm{A}$, where the $[\mathrm{O} \mathrm{I}] \lambda 6300$ emission peak is also located. Since [O I] $\lambda 6300$ traces shocks, this high $\sigma$ value is a hint of turbulence and shocks. The lowest velocity dispersion (lower limit $25 \pm 3 \mathrm{~km} \mathrm{~s}^{-1}$ ) corresponds to the area dominated by $\mathrm{H}$ II-like ionization, toward the east of the spiral arm (e.g., A7; see $\S 6.2$ ). This indicates that the velocity dispersion is likely dominated by the internal kinematics within the stellar clusters, rather than by large regions projected along the line of sight.

In this case it is not possible to state that there are clear indications of pure rotation, i.e., the photometric peak does not coincide with the velocity dispersion maximum, and neither of them coincides with the possible kinematic center. The velocity maps show that even if pure rotation does not dominate the galaxy kinematics, rotational movements can be present.

\subsubsection{NGC 3690}

As in the previous case, at a resolution of $0.18 \mathrm{kpc}$ the structure of the ionized and neutral gas velocity fields obtained with the SB2 bundle are very similar (Fig. 19 left and middle panels). The largest receding velocities are detected toward the northeast of the field $\left(\mathrm{C}^{\prime}\right.$ and $\left.\mathrm{C} 6\right)$, while the largest approaching velocities are located on B2 and toward the southwest. The ionized gas sys- temic velocity is defined as that of nucleus B1, with a value of $3040 \pm 27 \mathrm{~km} \mathrm{~s}^{-1}$. This value coincides with the Zhao et al. (1997) measurements using the $\mathrm{H} 92 \alpha$ radio recombination line $\left(3080 \pm 40 \mathrm{~km} \mathrm{~s}^{-1}\right)$. For the ionized gas, the peak-to-peak velocity is $257 \mathrm{~km} \mathrm{~s}^{-1}$. The systemic velocity measured with the neutral gas is $2976 \pm 32 \mathrm{~km} \mathrm{~s}^{-1}$, with a peak-to-peak variation of $217 \mathrm{~km} \mathrm{~s}^{-1}$. In both ionized and neutral gas, the velocity variations are similar to the ones measured in IC 694. As found in ULIRGs (Martin 2005), there is a systemic blueshift of the neutral gas velocity with respect to the ionized gas (about $-24 \pm$ $13 \mathrm{~km} \mathrm{~s}^{-1}$ in the studied regions). Due to its value, lower than the results founded for ULIRGs (from about -60 to $-300 \mathrm{~km} \mathrm{~s}^{-1}$; Martin 2005), this blueshift is regarded as a marginal effect.

Across the galaxy there are gradients of about $70 \mathrm{~km} \mathrm{~s}^{-1} \mathrm{kpc}^{-1}$ in the ionized gas (between $\mathrm{C}^{\prime}$ and $\mathrm{D} 3$ ). This result is close to the gradients derived for ULIRGs using a slit (up to $60 \mathrm{~km} \mathrm{~s}^{-1} \mathrm{kpc}^{-1}$ for an individual galaxy; Martin 2005).

The two-dimensional structure of the velocity dispersion ranges between $50 \pm 5$ and $172 \pm 20 \mathrm{~km} \mathrm{~s}^{-1}$ (Fig. 19, right panel) and presents different characteristics than the two-dimensional velocity fields. Nucleus B1 has the maximum velocity dispersion value of the entire system $\left(172 \pm 20 \mathrm{~km} \mathrm{~s}^{-1}\right)$, and regions with high velocity dispersion $\left(\sim 160 \mathrm{~km} \mathrm{~s}^{-1}\right)$ are also detected about $1 \mathrm{kpc}$ toward the north and northwest of B1. Looking at the ionized gas velocity field, these secondary peaks of the velocity dispersion are located in regions where there are high-velocity variations (from approaching to receding), and thus this $\sigma$ would represent a convolution of a large velocity gradient. Although the $\sigma$-peak coincides spatially with the nucleus, and then it would be tracing the mass (large $\sigma$ implies that this is the most massive galaxy of the system), the presence of an AGN in B1 could produce a broadening of the line width.

As in the case of IC 694, there is no clear evidence for ordered rotating motions; the photometric and velocity dispersion peaks do not coincide with the possible kinematic center. In conclusion, although the pure rotation does not dominate the kinematics of the galaxy, a rotational process would be present.

\subsection{Where Is the True Nucleus of NGC 3690?}

The galaxy NGC 3690 shows variations on the surface brightness importance of regions B1 and B2 with wavelength. While in 
the ultraviolet (Windhorst et al. 2002) and in the optical (this work) B2 appears brighter than B1, and therefore has been identified as the nucleus, the reverse is true in the near-IR $(2.2 \mu \mathrm{m}$; AAH00) and mid-IR (Gallais et al. 2004). Besides, the optical kinematical analysis shows that the $\sigma$ peak is located in B1 (see $\S 10.1 .3)$. This fact, along with the high extinction $\left(A_{V}=3.7\right)$, the hot dust component needed to explain the color excess, and the presence of an AGN, lead us to the conclusion that B1 is the true nucleus of the individual galaxy NGC 3690, as previously discussed by Gallais et al. (2004), who used mid-IR spectro-imaging, and as concluded by Zezas et al. (2003), who used Chandra X-ray observations.

\subsection{Dynamical Mass Derivation of the Nuclei and Brightest Regions}

Until now the only mass derivation in this work has been done for the $\mathrm{H} \alpha$-emitting young star-forming regions. The central ionized gas velocity dispersion is a good tracer of the dynamical mass; thus it can be used as an alternative method for mass derivation. Assuming virialization, the dynamical mass of the nuclei and brightest regions can be derived as

$$
M_{\mathrm{dyn}}=1.75 \times 10^{3} R_{\mathrm{hm}} \sigma^{2} M_{\odot},
$$

where $R_{\mathrm{hm}}$ is the half-mass radius in pc and $\sigma$ is the velocity dispersion in $\mathrm{km} \mathrm{s}^{-1}$. Assuming a constant value of 1.75 (see Colina et al. 2005 for a detailed discussion), and that the effective radius $\left(R_{\text {eff }}\right)$ of the light distribution traces the half-mass radius in each region, it is possible to infer the total dynamical mass.

In the present treatment we assume that $\mathrm{H} \alpha$ is traced by $\mathrm{Pa} \alpha$, which gives the real size of the emitting regions. In consequence, the effective radius of the most important emitting sources is calculated over the HST Pa $\alpha$ image; additional estimates using the F110M image are done for comparison. There are some caveats related to the spatial resolution of the different set of data worth mentioning. For this mass estimate we are dealing with information at different spatial resolutions. On the one hand, the velocity dispersion from the INTEGRAL SB2 bundle data was measured at a $0.9(\sim 180 \mathrm{pc})$ resolution. On the other hand, the $R_{\text {eff }}$ over the most relevant Arp 299 regions (i.e., the ones resolved) was measured using the NICMOS1/F110M image (0"043 pixel $^{-1}$, i.e., $\left.\sim 9 \mathrm{pc} \mathrm{pixel}^{-1}\right)$ and NICMOS2/Pa $\alpha$ image $\left(0.076\right.$ pixel $^{-1}$, i.e., $\sim 16 \mathrm{pc} \mathrm{pixel}^{-1}$ ). The typical size of the $\mathrm{H}$ II regions in LIRGs is 200-300 pc (Alonso-Herrero et al. 2002), i.e., several times larger than the effective radius of the stellar clusters and about the size of a SB2 fiber. Taking this into account, the clusters will be point sources for INTEGRAL, and their $\sigma$ will be integrated on the fiber.

For nucleus A we derive a dynamical mass of about $1.2 \times$ $10^{9} M_{\odot}$ (Table 10), which is about 5 times smaller than the derivation done by Shier et al. (1996) using the velocity dispersion measured from the $2.3 \mu \mathrm{m} \mathrm{CO}$ band; this discrepancy is due to a different estimate of the radius $\left(r_{\mathrm{CO}} / r_{\mathrm{Pa} \alpha} \simeq 4\right)$. The calculated $M_{\text {dyn }}$ implies that the young stellar mass content derived from $L(\mathrm{H} \alpha)$ in $\mathrm{A}$ is about $40 \%$. The mean value for the dynamical mass of nucleus B1 is $1.5 \times 10^{9} M_{\odot}$ (in good agreement with Shier et al. 1996); consequently, the upper limit for the young stellar mass content is about $15 \%$. For B2 the mean value of the total mass is $0.6 \times 10^{9} M_{\odot}$ (the same as Shier et al. 1996). This would imply that about $100 \%$ of the mass in B2 is from young stellar population, indicating that the mass derivation done using $L(\mathrm{H} \alpha)$ is probably overestimated. For the last two regions ( $\mathrm{C} 1$ and $\mathrm{C}^{\prime}$ ), the $R_{\mathrm{eff}}$ gives an upper limit for the dy-
TABLE 10

Dynamical Mass Eestimates

\begin{tabular}{|c|c|c|}
\hline Region & $\begin{array}{l}R_{\mathrm{eff}}^{\mathrm{a}} \\
(\mathrm{kpc})\end{array}$ & $\begin{array}{c}M_{\mathrm{dyn}}^{\mathrm{b}} \\
\left(\times 10^{9} M_{\odot}\right)\end{array}$ \\
\hline ........ & $0.054-0.062$ & $1.10-1.3$ \\
\hline В1 & $0.035-0.022$ & $1.81-1.1$ \\
\hline В2 & $0.030-0.025$ & $0.63-0.5$ \\
\hline $\mathrm{C} 1$ & $0.022-0.042$ & $0.33-0.6$ \\
\hline $\mathrm{C}^{\prime}$ & $0.023-0.043$ & $0.19-0.3$ \\
\hline
\end{tabular}

Notes.-Dynamical mass estimates for the nuclei of the system (A and $\mathrm{B} 1$ ) and main $\mathrm{H} \alpha$-selected emitting regions (B2, C1, and $\left.\mathrm{C}^{\prime}\right)$.

${ }^{a}$ The stellar and gas effective radius have been derived for the HST F110M (first value) and for F190N ( $\mathrm{Pa} \alpha$ ) (second value).

${ }^{\mathrm{b}}$ For both $R_{\text {eff }}$ the dynamical mass has been derived using the $\mathrm{H} \alpha$ velocity dispersion values. See $\S 10.3$ for details.

namical mass $\left(0.6\right.$ and $0.3 \times 10^{9} M_{\odot}$, respectively, the latter according to the $>0.1 \times 10^{9} M_{\odot}$ from AAH00) that implies young stellar mass contents of about $13 \%$ and $40 \%$, respectively.

\section{SUMMARY}

A detailed IFS study of the LIRG Arp 299 (IC 694 and NGC 3690 ) system covering a region of $7.0 \times 6.2 \mathrm{kpc}$ has been presented. This work illustrates the flexibility and power of the IFS in studying in two dimensions and simultaneously the different physical properties, such as stellar populations, internal dust extinction, ionization structure, and gas kinematics, of complex systems like Arp 299. In what follows only the main results of this study are summarized:

1. The observed overall two-dimensional stellar and ionized gas structure differs due to internal extinction effects caused by the dust, and to the different spatial distribution of the diverse (AGNs, stars, shocks) ionizing sources. The stellar continuum and emission-line distribution peaks are not spatially coincident but separated up to $1.4 \mathrm{kpc}$. The peaks of the ionized gas traced by different emission lines (e.g., hydrogen recombination, high excitation [O III], and shock-tracer [O I ]) are also not coincident, indicating that the ionizing mechanisms change on scales of about 200 pc or less.

2. Region B1 is identified as the true nucleus of NGC 3690 . The presence of an AGN in B1 is inferred from the detection of a high-excitation Seyfert-like conical structure of about $1.5 \mathrm{kpc}$ in size with an opening angle of about $54^{\circ}$ and its apex located in $\mathrm{B} 1$. This region also presents the additional characteristics of a dust-enshrouded nucleus with high extinction $\left(A_{V}=3.7 \pm 0.6\right)$, red colors $(\mathrm{F} 160 \mathrm{~W}-\mathrm{F} 222 \mathrm{M}=2.06 \pm 0.1)$ indicating hot dust emission, and high velocity dispersion $\left(\sigma=172 \pm 20 \mathrm{~km} \mathrm{~s}^{-1}\right)$.

3. The observed near-IR colors reveal the presence of hot dust over the entire structure. According to the F160W - F222M color, the upper limit for the mean hot dust contribution is about $30 \%$ at $600 \mathrm{~K}$ for the majority of the star-forming regions. Nuclei $\mathrm{A}$ and $\mathrm{B} 1$ and region $\mathrm{C} 1$ are the most affected by the hot dust component, with upper limits of about $70 \%, 80 \%$, and $90 \%$ at $600 \mathrm{~K}$, respectively.

4. The high surface brightness regions are mainly dominated by star formation, while the low surface brightness regions are dominated by LINER-like ionization process. The $\mathrm{H} \alpha$-emitting star-forming regions present an extinction of between $A_{V}=1-$ 6 mag, with ages between 3.3 and $7.2 \times 10^{6} \mathrm{yr}$, and masses that range between about 6 and $680 \times 10^{6} M_{\odot}$. The total contribution (extinction corrected) of these sources to the bolometric luminosity is about $45 \%$. 
5. The structure of the ionized $(\mathrm{H} \alpha)$ and neutral $(\mathrm{Na} \mathrm{D})$ gas two-dimensional velocity fields show similar features, indicating that both components are kinematically coupled on scales of hundreds of parsecs and larger. The velocity fields are very complex, and in general the galaxy does not appear to be dominated by ordered virialized simple (e.g., rotation) motions. The morphology of the velocity field does not correlate with the distribution of mass as traced by the continuum images, that is, no spatial coincidence between the kinematic and the photometric centers exists.

On average, the interface region presents the lowest value of velocity dispersion $\left(\sigma \sim 45 \mathrm{~km} \mathrm{~s}^{-1}\right)$, IC 694 presents the intermediate values $\left(\sigma \sim 65 \mathrm{~km} \mathrm{~s}^{-1}\right)$, and NGC 3690 the highest $\left(\sigma \sim 120 \mathrm{~km} \mathrm{~s}^{-1}\right)$. This indicates that NGC 3690 is the warmest body of the entire system, and probably the most massive. On the other hand, the low velocity amplitude and velocity dispersion of the interface suggests that the ionized gas is slowly rotating or in a close quiescent phase.

The velocity dispersion measurements over the nuclei and the brightest emitting regions, imply a mean value for the dynamical mass of about $1.2 \times 10^{9} M_{\odot}$ and $1.5 \times 10^{9}$ for nuclei A and B1, respectively, within a mean radius of 58 and $28 \mathrm{pc}$. For regions $\mathrm{B} 2, \mathrm{C} 1$, and $\mathrm{C}^{\prime}$ the mass ranges between $\sim 0.2 \times 10^{9} M_{\odot}$ and $\sim 0.6 \times 10^{9} M_{\odot}$, within a radius of about $30 \mathrm{pc}$.

The authors wish to thank Hajime Sugai, the referee, for his detailed and constructive report. We thank Jesús Maíz-Apellániz for his help using jmaplot. This work has been supported by the Spanish Ministerio de Educación y Ciencia, under grant BES2003-0852, projects AYA2002-01055 and ESP2005-01480.
Aller, L. H. 1984, Physics of Thermal Gaseous Nebulae (Dordretch: Reidel) Alonso-Herrero, A., Rieke, G. H., Rieke, M. J., Colina, L., Pérez-González, P. G., \& Ryder, S. D 2006, ApJ, 649, in press

Alonso-Herrero, A., Rieke, G. H., Rieke, M. J., \& Scoville, N. Z. 2000, ApJ, 532, 845 (AAH00)

- 2002, AJ, 124, 166

Armus, L., Heckman, T. M., \& Miley, G. K. 1989, ApJ, 347, 727

Arribas, S., Mediavilla, E., García-Lorenzo, B., \& del Burgo, C. 1997, ApJ, 490, 227

Arribas, S., et al. 1998, Proc. SPIE, 3355, 821

Augarde, R., \& Lequeux, J. 1985, A\&A, 147, 273

Baan, W. A., \& Haschick, A. 1990, ApJ, 364, 65

Baldwin, J. A., Phillips, M. M., \& Terlevich, R. 1981, PASP, 93, 5

Ballo, L., Braito, V., Della Ceca, R., Maraschi, L., Tavecchio, F., \& Dadina, M. 2004, ApJ, 600, 634

Bingham, R. G., Gellatly, D. W., Jenkins, C. R., \& Worswick, S. P. 1994, Proc. SPIE, 2198, 56

Calzetti, D., Armus, L., Bohlin, R. C., Kinney, A. L., Koornneef, J., \& Storchi-Bergmann, T. 2000, ApJ, 533, 682

Calzetti, D., et al. 2005, ApJ, 633, 871

Charmandaris, V., Stacey, G. J., \& Gull, G. 2002, ApJ, 571, 282

Colina, L., Arribas, S., \& Borne, K. D. 1999, ApJ, 527, L13

Colina, L., Arribas, S., \& Monreal-Ibero, A. 2005, ApJ, 621, 725

Coziol, R., Torres, C. A. O., Quast, G. R., Contini, T., \& Davoust, E. 1998, ApJS, 119, 239

Della Ceca, R., et al. 2002, ApJ, 581, L9

Dopita, M. A., \& Sutherland, R. S. 1995, ApJ, 455, 468

Gallais, P., Charmandaris, V., Le Floc'h, E., Mirabel, I. F., Sauvage, M., Vigroux, L., \& Laurent, O. 2004, A\&A, 414, 845

García-Lorenzo, B., Acosta-Pulido, J. A., \& Megias-Fernández, E. 2002, in ASP Conf. Ser. 282, Galaxies: The Third Dimension, ed. M. Rosado, L. Binette, \& L. Arias (San Francisco: ASP), 501

Gehrz, R. D., Sramek, R. A., \& Weedman, D. W. 1983, ApJ, 267, 551

Heckman, T. M., Armus, L., \& Miley, G. K. 1990, ApJS, 74, 833

Hibbard, J. E., \& Yun, M. S. 1999, AJ, 118, 162

Hildebrand, R. H. 1983, QJRAS, 24, 267

Holtzman, J. A., et al. 1995, PASP, 107, 156

Howarth, I. D., \& Murray, J. 1988, DIPSO A Friendly Spectrum Analysis Program (Starlink User Note 50; Chilton: Rutherford Appleton Lab)
Keel, W. C. 1984, ApJ, 282, 75

Kennicutt, R. C. 1998, ARA\&A, 36, 189

Keto, E., et al. 1997, ApJ, 485, 598

Laurent, O., Mirabel, I. F., Charmandaris, V., Gallais, P., Madden, S. C., Sauvage, M., Vigroux, L., \& Cesarsky, C. 2000, A\&A, 359, 887 Leitherer, C., et al. T. M. 1999, ApJS, 123, 3

McLeod, B. 1997, in The HST Calibration Workshop, ed. S. Casertano, R. Jederzejewski, C. D. Keyes, \& M. Stevens (Baltimore: STSciI), 281

Mannucci, F., et al. 2003, A\&A, 401, 519

Martin, C. L. 2005, ApJ, 621, 227

Mattila, S., et al. 2005, IAU Circ., 8473, 1

Mihos, J. C., \& Hernquist, L. 1994, ApJ, 431, L9

Monreal-Ibero, A., Arribas, S., \& Colina, L. 2006, ApJ, 637, 138

Neff, S. G., Ulvestad, J. S., \& Teng, S. H. 2004, ApJ, 611, 186

Origlia, L., \& Leitherer, C. 2000, AJ, 119, 2018

Osterbrock, D. E. 1989, Soviet Astron., 33, 694

Rampazzo, R., Plana, H., Amram, P., Bagarotto, S., Boulesteix, J., \& Rosado, M. 2005, MNRAS, 356, 1177

Sanders, D. B., \& Mirabel, I. F. 1996, ARA\&A, 34, 749

Satyapal, S., Watson, D. M., Pipher, J. L., Forrest, W. J., Fischer, J., Greenhouse,

M. A., Smith, H. A., \& Woodward, C. E. 1999, ApJ, 516, 704

Savage, B. D., \& Mathis, J. S. 1979, ARA\&A, 17, 73

Scoville, N. Z., et al. 2000, AJ, 119, 991

Shier, L. M., Rieke, M. J., \& Rieke, G. H. 1996, ApJ, 470, 222

Sparks, W. B., Carollo, C. M., \& Macchetto, F. 1997, ApJ, 486, 253

Stanford, S. A., \& Wood, D. O. S. 1989, ApJ, 346, 712

Sugai, H., Davies, R. I., Malkan, M. A., McLean, I. S., Usuda, T., \& Ward, M. J. 1999, ApJ, 527, 778

Telesco, C. M., Decher, R., \& Gatley, I. 1985, ApJ, 299, 896

van den Bergh, S. 1995, Nature, 374, 215

Vazquez, G. A., \& Leitherer, C. 2005, ApJ, 621, 695

Veilleux, S., \& Osterbrock, D. E. 1987, ApJS, 63, 295

Windhorst, R. A., et al. 2002, ApJS, 143, 113

Zezas, A., Ward, M. J., \& Murray, S. S. 2003, ApJ, 594, L31

Zhao, J., Anantharamaiah, K. R., Goss, W. M., \& Viallefond, F. 1997, ApJ, 482, 186 
The Astrophysical Journal, 654:696, 2007 January 1

(C) 2007. The American Astronomical Society. All rights reserved. Printed in U.S.A.

\section{ERRATUM: "INTEGRAL FIELD SPECTROSCOPY OF THE LUMINOUS INFRARED}

GALAXY ARP 299 (IC 694 + NGC 3690)” (ApJ, 650, 850 [2006])

M. García-Marín, L. Colina, S. Arribas, A. Alonso-Herrero, and E. Mediavilla

Due to an error at the Press, the header to column (8) of Table 1 is incorrect. This column head should read log $([\mathrm{O}$ I $] / \mathrm{H} \alpha)$, not $\log ([\mathrm{O}$ III $/ \mathrm{H} \alpha)$.

The Press sincerely regrets this error. 